Radiation Physics and Chemistry 70, (2004), 323-344

ISSN: 0969-806X

doi: 10.1016/j.radphyschem..2003.12.019

(C) 2003 Elsevier Ltd. All rights reserved.

http://www.elsevier.com/wps/find/homepage.cws home

http://www.elsevier.com/locate/radphyschem

\title{
Self-consistent R-matrix approach to photoionization and unified electron-ion recombination
}

\author{
Sultana N. Nahar, Anil K. Pradhan \\ Department of Astronomy, The Ohio State University, Columbus, OH 43210, USA
}

\begin{abstract}
A unified scheme using the R-matrix method has been developed for electron-ion recombination subsuming heretofore separate treatments of radiative and dielectronic recombination (RR and DR). The ab initio approach within the coupled channel approximation has several inherent advantages in addition to the natural unification of resonant and non-resonant phenomena. It enables a general and self-consistent treatment of photoionization and electron-ion recombination employing identical wavefunction expansion. Detailed balance takes account of interference effects due to resonances in cross sections, calculated explicitly for a large number of recombined $(e+$ ion) bound levels over extended energy regions. The theory of DR by Bell and Seaton is adapted for high- $n$ resonances in the region below series limits. The R-matrix method is employed for (A) partial and total photoionization and photorecombination cross sections of $(\mathrm{e}+$ ion $)$ bound levels, and (B) DR and $(\mathrm{e}+$ ion) scattering cross sections. Relativistic effects and fine structure are considered in the Breit-Pauli approximation. Effects such as radiation damping may be taken into account where necessary. Unified recombination cross sections are in excellent agreement with measurements on ion storage rings to about $10-20 \%$. In addition to high accuracy, the strengths of the method are: (I) both total and level-specific cross sections and rate coefficients are obtained, and (II) a single $(\mathrm{e}+$ ion) recombination rate coefficient for any given atom or ion is obtained over the entire temperature range of practical importance in laboratory and astrophysical plasmas, (III) self-consistent results are obtained for the inverse processes of photoionization and recombination; comprehensive datasets have been computed for over 50 atoms and ions. Selected data are presented for iron ions.
\end{abstract}

\section{Introduction}

The electron-ion recombination process is unified in nature. It involves both resonant and non-resonant components that are inseparable in principle and always occur together, analogous to those in the complementary physical processes of electron-ion scattering and photoionization. Experiments or observations of $(\mathrm{e}+$ ion) recombination measure the total cross section. Theoretically, therefore a unified treatment with a self-consistent approach is preferable to methods that consider $(\mathrm{e}+\mathrm{ion})$ recombination in parts employing different approximations of varying validity.

Historically, $(\mathrm{e}+$ ion) recombination is usually considered in two main but separate parts: (a) radiative recombination $(\mathrm{RR})$ i.e. direct radiative capture and recombination,

$$
\mathrm{e}+X^{n+} \rightarrow h v+X^{(n-1)+}
$$


and dielectronic recombination (DR) i.e. indirect capture and recombination through autoionizing states,

$$
\mathrm{e}+X^{n+} \rightarrow X^{(n-1)+* *} \rightarrow h v+X^{(n-1)+} .
$$

where the intermediate state (indicated by double asterisks) is a doubly excited autoionizing state of the $(\mathrm{e}+\mathrm{ion})$ system which introduces a resonance.

The subject of electron-ion recombination has been one of the most active areas of research in atomic physics for several decades, both theoretically and experimentally. Traditionally the two parts, RR and DR, are treated independently. The RR cross sections are obtained in a straightforward manner using detailed balance (Milne relation) from ground state photoionization cross sections computed using relatively simple approximations such as the central field or the quantum defect method (Burgess and Seaton, 1960), without taking account of resonances. On the other hand the theoretical treatment of DR has a long and interesting history (Seaton and Storey, 1976; Hahn and Lagattuta, 1988). The main development was the realization by Burgess (1965) that DR via the infinite series of resonances in the $(e+$ ion) system is an important contributor to the total recombination process. The celebrated general Burgess formula was used in many applications, particularly in astrophysical modeling. More accurate treatments of DR, generally based on the isolated resonance approximation using the distorted wave method, were later developed taking account of physical effects not included in the Burgess formula, such as autoionization into excited levels (e.g. Jacobs et al., 1977). Nussbaumer and Storey (1983) pointed out the importance of low-energy resonances that might give significant enhancement of the DR rate in the low-temperature region. Hahn (1985) provided expressions for DR cross sections for comparison with experiments. There are many calculations for dielectronic recombination (DR) with highly charged ions (e.g. Pindzola et al., 1990; Badnell et al., 1990), using not only the distorted wave method also others such as the saddle point variation method (Mannervik et al., 1998), that yield good agreement with experimental data for the ions considered.

In recent years, a number of pioneering experimental studies have been carried out. Experimental measurements of electron-ion recombination cross sections using ion storage rings exhibit detailed resonance structures at very high resolution in beam energy (e.g. Wolf et al., 1991; Kilgus et al., 1990, 1993; Mannervik et al., 1998; Schippers et al., 1999). The experiments measure absolute cross sections and therefore provide ideal tests for theoretical methods, as well as the physical effects included in the calculations. In light of the new experimental studies however, and given that the unified method for electron-ion recombination is quite general, it is desirable to extend the calculations to elicit detailed features for direct comparison with the measured cross sections. One of the goals of the present article is to demonstrate the accuracy of the method, on par with the R-matrix treatment of photoionization and electron impact excitation, as well as to study theoretical issues such as relativistic effects, the distinction between close coupling and independent resonance treatments, the magnitude of the resonant and the non-resonant (background) cross sections, relatively sparse near-threshold resonance structures as opposed to the dense resonances below the Rydberg series limits, radiation damping of low-lying autoionizing resonances, etc.

In the present work we describe photoionization, electron-ion scattering, and (e + ion) recombination self-consistently within the framework of the close coupling approximation using the R-matrix method. Combining Eqs. (1) and (2), and invoking detailed balance, we may write 
$\mathrm{e}+X^{n+} \leftrightarrow X^{(n-1)+* *} \leftrightarrow h v+X^{(n-1)+}$,

where photoionization and recombination (resonant $\oplus$ non-resonant) proceed inversely in either direction. The wavefunctions for the $(\mathrm{e}+\mathrm{ion})$ system are obtained with the same eigenfunction expansion for both processes, enabling a self-consistent treatment in an ab initio manner. The R-matrix package of codes has been extended to incorporate the theoretical framework described below.

\section{Theoretical framework}

The R-matrix method developed by Burke and associates (Burke and Robb, 1975; Burke and Berrington, 1993) provides a natural and powerful tool for collisional and radiative electron-ion processes based on the close coupling or coupled channel (CC) approximation. In particular, the R-matrix method has been employed for large-scale computations using efficient codes developed under the Opacity project (The Opacity Project, 1995/1996; Seaton, 1987;

Berrington et al., 1987), and the Iron Project (Hummer et al., 1993; Berrington et al., 1995). Most of these calculations entail photoionization and electron-ion scattering, the two processes that form the basis of the present extension of the R-matrix method to unified ( $\mathrm{e}+\mathrm{ion}$ ) recombination (Nahar and Pradhan, 1992, 1994a, 1995, 1997; Zhang and Pradhan, 1997; Zhang et al., 1999).

In the following subsections we describe the basic theory.

\subsection{Coupled channel wavefunction and radiative transitions}

The total wavefunction for a $(N+1)$-electron $(\mathrm{e}+$ ion) system in the CC approximation is described as

$$
\Psi_{E}(\mathrm{e}+\text { ion })=A \sum_{j} \chi_{i}(\text { ion }) \theta_{i}+\sum_{i} c_{j} \Phi_{j}(\mathrm{e}+\text { ion })
$$

where $\chi_{i}$ is the target ion or core wavefunction in a specific state $S_{i} L_{i} \pi_{i}$ or level $J_{i} \pi_{i}$; $\theta_{i}$ is the wavefunction of the interacting $(N+1)$ th electron in a channel labeled as $S_{i} L_{i}\left(J_{i}\right) \pi i k_{i}^{2} \ell_{i}(S L \pi$ or $J \pi) ; k_{i}^{2}$ is the incident kinetic energy. $\Phi_{j}$ is the correlation functions of $(\mathrm{e}+$ ion) system that compensates the orthogonality condition and short-range correlation interactions. The target wavefunctions are obtained from atomic structure treatment (e.g. Eissner et al., 1974). The complex resonant structures in photoionization, recombination, and in electron impact excitation are included through channel couplings.

Relativistic effects are included through Breit-Pauli approximation in intermediate coupling. The $(N+1)$-electron Hamiltonian in the Breit-Pauli R-matrix (BPRM) method, as adopted in the Iron project (Hummer et al., 1993), is

$$
H_{N+1}^{\mathrm{BP}}=H_{N+1}^{\mathrm{NR}}+H_{N+1}^{\mathrm{mass}}+H_{N+1}^{\mathrm{Dar}}+H_{N+1}^{\mathrm{so}},
$$

where non-relativistic Hamiltonian is

$$
H_{N+1}^{\mathrm{NR}}=\sum_{i=1}^{N+1}\left\{-\nabla_{i}^{2}-\frac{2 Z}{r_{i}}+\sum_{i>i}^{N+1} \frac{2}{r_{i j}}\right\}
$$


$H_{\underline{N}+1}^{\text {nass }}=-\left(\alpha^{2} / 4\right) \sum_{i} p_{i}^{4}$, is the mass correction term, $H_{N+1}^{\text {Dar }}=\left(Z \alpha^{2} / 4\right) \sum_{i} \nabla^{2}\left(1 / r_{i}\right)$ is the Darwin term, and $\left.H_{N+1}^{\text {so }}=Z x^{i} \sum_{i}\left(1 / r_{i}^{5}\right)\right]_{i} \mathbf{s}_{i}$ is the spin-orbit interaction term. Spin-orbit interaction splits the LS terms into fine-structure levels labeled by $J \pi$ where $J$ is the total angular momentum. Solutions of the Schrodinger equation, $H_{N+1}^{\mathrm{RP}} \Psi=E \Psi$ which becomes a set of coupled equations with the CC expansion, give the bound wavefunctions, $\Psi_{\mathrm{B}}$; for negative energies $(E<0)$; and continuum wavefunction, $\Psi_{\mathrm{F}}$; for positive energies $(E \geq 0)$ :

The transition matrix elements for various atomic processes are: $\left\langle\Psi_{\mathrm{B}}\|\mathrm{D}\| \Psi_{\mathrm{F}}\right\rangle$ for photoionization and recombination, $\left\langle\Psi_{\mathrm{B}_{\mathrm{B}}}\|\mathbf{D}\| \Psi_{\mathrm{B}^{\prime}}\right\rangle$ for oscillator strength, $\left\langle\Psi_{\mathrm{F}}\right| H(e+$ ion $)\left|\Psi_{\mathrm{F} .}\right\rangle$ for electron impact excitation, where $\mathbf{D}$ is the dipole operator, $\mathbf{D}_{\mathrm{L}}=\sum_{\mathrm{i}} r_{i}$ in "length" form and $\mathbf{D}_{\mathrm{V}}$. $=-2 \sum_{i} \Delta_{i}$ in "velocity" form with the sum over the number of electrons. The matrix elements are divided into inner and outer region components corresponding to the R-matrix boundary at a suitably chosen radius $(r=a)$; and asymptotic wavefunctions for $r \rightarrow \infty$ :

The transition matrix element with the dipole operator can be reduced to the generalized line strength defined, in either length or velocity form, as

$$
\begin{aligned}
& S_{\mathrm{L}}=\left|\left\langle\Psi_{\mathrm{f}}\left|\sum_{j=1}^{N+1} r_{j}\right| \Psi_{\mathrm{i}}\right\rangle\right|^{2}, \\
& S_{\mathrm{V}}=\omega^{-2}\left|\left\langle\Psi_{\mathrm{f}}\left|\sum_{j=1}^{N+1} \frac{\partial}{\partial r_{j}}\right| \Psi_{i}\right\rangle\right|^{2},
\end{aligned}
$$

where $\omega$ is the incident photon energy in Rydberg units, and $\Psi_{\mathrm{i}}$ and $\Psi_{\mathrm{f}}$ are the wavefunctions representing the initial and final states, respectively.

The photoionization cross section $\left(\sigma_{\mathrm{PI}}\right)$ is proportional to the generalized line strength $(S)$;

$$
\sigma_{\mathrm{PI}}=\frac{4 \pi}{3 c} \frac{1}{g_{i}} \omega S
$$

where $g_{i}$ is the statistical weight factor of the initial state.

\subsection{Electron-ion recombination—detailed balance}

The total recombination cross section is the sum of cross sections for recombination into the infinite number of recombined levels of the $(\mathrm{e}+\mathrm{ion})$ system, as illustrated schematically in Fig. 1.

We divide this infinite set into 3 groups: (i) recombination into low- $n$ levels with $n \leq n_{o}$; (ii) contribution from high- $n$ resonances to bound levels with $n_{o} \leq n \leq \infty$ (DR only), and (iii) “top-up" background contribution from $n>n_{o}$ (RR only). The $n_{o}$ is chosen such that all three subsets are independent and complementary. The contributions to recombination from states with $n \leq n_{o}$ (i) are obtained from detailed $\sigma_{\text {PI }}$; typically with extensive resonance structures, using the principle of detailed balance (Milne relation). The recombination cross section, $\sigma_{\mathrm{RC}}$; is related to photoionization cross section, $\sigma_{\mathrm{PI}}$; through the principle of detailed balance,

$$
\sigma_{\mathrm{RC}}=\sigma_{\mathrm{PI}} \frac{g_{i}}{g_{j}} \frac{h^{2} \omega^{2}}{4 \pi^{2} m m^{2} c^{2} v^{2}}
$$

The recombination rate coefficient, $\alpha_{\mathrm{RC}}$; is obtained as 


$$
\alpha_{\mathrm{RC}}(T)=\int_{0}^{\infty} v f(v) \sigma_{\mathrm{RC}} \mathrm{d} v
$$

where $f(v)$ is the Maxwellian velocity distribution function. The total $\alpha_{\mathrm{RC}}$ is obtained from contributions from infinite number of recombined states as

$$
\begin{aligned}
\alpha_{\mathrm{R}}(T)= & \sum_{i_{b}} \frac{g_{i}}{g_{j}} \frac{2}{k T \sqrt{2 \pi m^{3} k c^{2} T}} \\
& \times \int_{0}^{\infty} E^{2} \sigma_{\mathrm{PI}}\left(i_{b} ; z\right) \mathrm{e}^{-\varepsilon / k T} \mathrm{~d} z,
\end{aligned}
$$

where $E=h \omega=\varepsilon+I_{p}, \varepsilon$ is the photoelectron energy, and $I_{\mathrm{p}}$ is the ionization potential. The integration over $\infty$ energy range of the photoelectron is carried out as described in Nahar and Pradhan (1994a). $\sigma_{\mathrm{PI}}$; obtained including the autoionizing resonances, essentially provide total $\alpha_{R}(T)$ incorporating both the RR and the DR in a unified manner.

\subsection{Dielectronic recombination via high-n resonances}

Recombination into the high- $n$ levels of the $(\mathrm{e}+\mathrm{ion})$ system via resonances with $n_{o}<n \leq$ $\infty$ approaching target ions series limits, (process (3) in Fig. 1) is included by implementing the extension of precise theory of DR

Ion States (Continua)

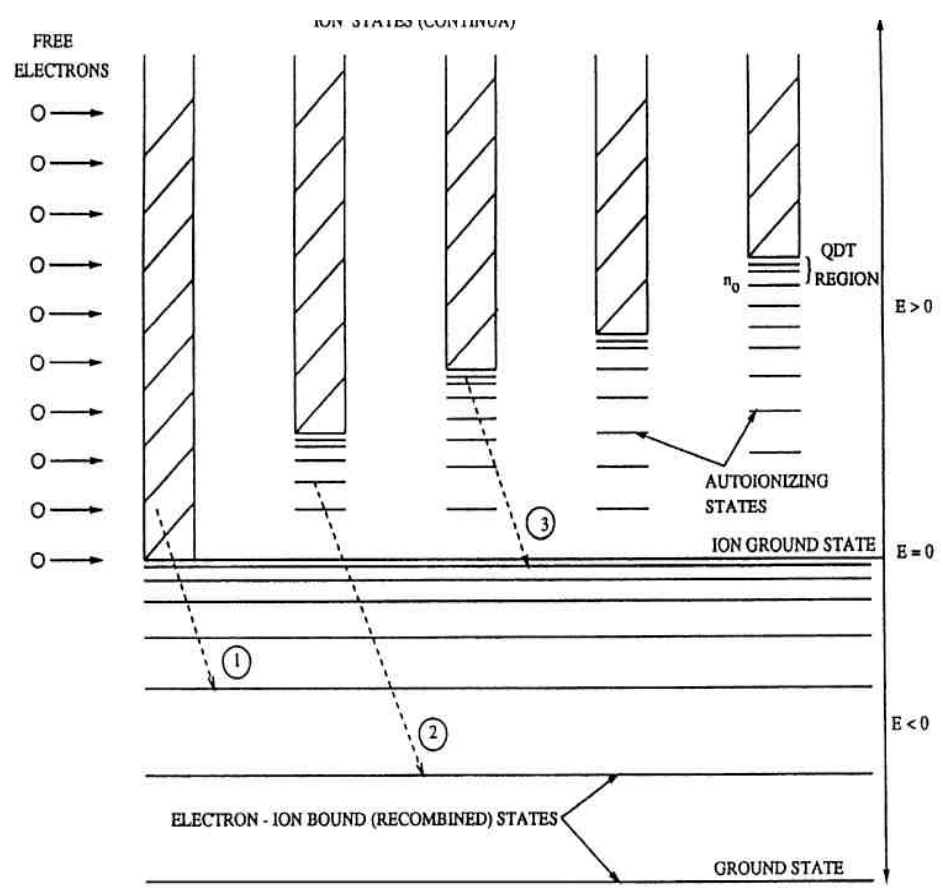

Fig. 1. Schematic energy diagram of unified $(e+$ ion) recombination. The infinite series of autoionizing resonances converging onto the various excited target states are in the positive energy region $E>0$, while recombined states are in the negative energy region $E<0$. Broken lines with arrows represent photon emission during recombination: (1) recombination through the ground state continuum of the recombined ion, (2) through a low- $n$ autoionizing state with possibly large interaction with the continua (coupling to excited states gives rise to autoionizing resonances), and (3) through a high-autoionizing state, with negligible continuum contribution (DR) only. 
by Bell and Seaton (hereafter BS, 1985, as adapted in Nahar and Pradhan, 1994a, 1995) based on the CC approximation. To each excited threshold $S_{i} L_{i}\left(J_{i}\right) \pi_{i}$ of the $N$-electron target ion, there corresponds an infinite series of $(N+1)$-electron states, $S_{i} L_{i}\left(J_{i}\right) \pi_{i} v \ell$, to which recombination can occur, where $v$ is the effective quantum number of the autoionizing level. The recombining electron is treated as a 'spectator', recombining from an autoionizing level $v \ell$ to the corresponding $n \ell$ bound level following the ion core transition. For sufficiently high- $n$ DR dominates the recombination process and the background recombination is negligibly small. The contributions from these states are added by calculating the collision strengths, $\Omega_{\mathrm{DR}}$. Several aspects related to the application of the theory to the calculation of DR collision strengths are described in Nahar and Pradhan (1992, 1994a, 1995), Nahar (1996b), Zhang et al. (1999). We sketch below a few working expressions derived from the theory.

Including radiative interactions in an ab initio manner in the total Hamiltonian for the e + ion system, a generalized electron-photon scattering matrix $\mathscr{S}$ may be obtained as (Davies and Seaton, 1969)

$$
\mathscr{S}=\left(\begin{array}{ll}
\mathscr{S}_{\mathrm{ee}} & \mathscr{S}_{\mathrm{ep}} \\
\mathscr{S}_{\mathrm{pe}} & \mathscr{I}_{\mathrm{pp}}
\end{array}\right),
$$

where $\mathscr{S}$ ee is the matrix for electron scattering including radiation damping; $\mathscr{S}$ pe is the matrix for electron capture followed by radiative decay with the emission of a photon; $\mathscr{S}$ ep that for the inverse process of photoionization; and $\mathscr{S}$ pp for photon-photon scattering. In the absence of interaction with the radiation field $\mathscr{S}$ ee is the usual scattering matrix $\mathbf{S}$. The unitarity condition for (electron + photon) $\mathscr{S}$ matrix reflects the conservation of both the incident electron and the emitted photon flux (Davies and Seaton, 1969), i.e.

$$
\mathscr{S}_{\mathrm{ee}}^{\hat{\mathscr{T}}} \mathscr{S}_{\mathrm{ec}}+\mathscr{H}_{\mathrm{pe}}^{\dagger} \mathscr{S}_{\mathrm{pe}}=1 \text {. }
$$

The electron-electron scattering matrix, $\mathscr{S}$ ee; may be again partitioned into sub-matrices of open and closed channels, in the energy region below threshold, in terms of its analytic continuation given by the matrix $\chi$ as $\chi_{\text {oo }}, \chi_{\text {oc }}, \chi_{\text {co, }}$ and $\chi_{\text {cc }}$, where ' $o$ ' denotes the open and ' $c$ ' the closed channels. The open channels are those that are accessible to the incident electron for excitation of a target state in that channel; a closed channel refers to electron energies below an inaccessible target threshold. A given Rydberg series of resonances, converging on to a target

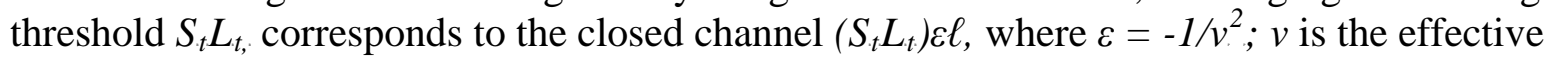
quantum number associated with the resonance series. The scattering matrix, $\mathscr{I}$ ee; is then obtained as (BS 1985)

$$
\mathscr{Y}_{\mathrm{ee}}=\chi_{\mathrm{oo}}-\chi_{\mathrm{oc}}\left[\chi_{\mathrm{cc}}-g(v) \exp (-2 \mathrm{i} \pi v)\right]^{-1} \chi_{\mathrm{co}}
$$

where $g(v)=\exp \left(\pi v^{3} \Gamma^{\mathrm{r}} / \mathrm{z}^{2}\right) ; \Gamma^{\mathrm{r}}$ is the sum of all possible radiative decay probabilities for the resonance series. These decay probabilities correspond to radiative transitions within the ion core. The outer electron is treated as a "spectator", in a high- $n$ resonance state, interacting but weakly with the core.

The electron flux trapped in the closed channel resonances may decay radiatively to bound states of the e+ion system. In multi-channel quantum defect theory we diagonalize the $\chi$. matrix as $\chi_{\text {cc }} \boldsymbol{N}=\mathbf{N} \chi_{\text {cc }}$; where $\chi_{\text {сc }}$ is a diagonal matrix and $\boldsymbol{N}$ is the diagonalizing matrix with $\boldsymbol{N}^{T} \boldsymbol{N}=\mathbf{1}$. In 
terms of $\boldsymbol{N}$ we write $\chi_{\text {oc }}^{\prime}=\chi_{\text {oc }} \boldsymbol{N}$ and $\chi_{\text {co }}^{{ }_{\text {co }}}=\boldsymbol{N}^{\mathrm{T}}{ }_{\chi \text { co }}$; where $\boldsymbol{N}^{\mathbf{T}}$, is the transpose of $\mathbf{N}$. The DR probability, for an entrance or incident open channel $\alpha$; is obtained from the unitarity condition as

$$
P_{x}(\mathrm{DR})=\left(\mathbf{1}-\mathscr{P}_{\mathrm{ee}}^{\dagger} \mathscr{S}_{\mathrm{ee}}\right)_{x \times}
$$

Substituting the proper expressions, the DR probability can be written as (Nahar and Pradhan, 1994a; Nahar, 1996b)

$$
\begin{aligned}
& P_{\alpha}=G(v) \sum_{\gamma}\left\{\left(\sum_{y^{\prime}} \chi_{\alpha \gamma^{\prime}}^{\prime} \mathbf{N}_{\gamma^{\prime}}\right)\left[\frac{1}{\chi_{\gamma^{\prime}}-g(v) \exp (-2 \pi \mathrm{i} v)}\right]\right. \\
& \left.\times\left[\frac{1}{x_{w^{*}}^{*}-g(v) \exp (+2 \pi \mathrm{iv})}\right]\left(\sum_{y^{\prime}} \chi_{\gamma^{\prime} \alpha}^{\prime *} \mathbf{N}_{F^{\prime}}^{*}\right)\right\},
\end{aligned}
$$

where $G(v)=g(v)^{2}-1=\exp \left(2 \pi v^{3} \Gamma^{\mathrm{r}} / z^{2}\right)-1$. The summations go over the closed channels $y y^{\prime}$ contributing to DR. The sum over the diagonal elements of all open channels linked to the ground state of the target ion gives the probability of DR through radiative transitions between the excited states and the ground state. As we are interested only in the detailed DR collision strengths, expressions derived for resonance averaged DR collision strengths, that are useful in the calculation of recombination rate coefficients, are not given here but may be found in Nahar and Pradhan (1994a) and Nahar (1996b).

The DR collision strength, $\Omega(\mathrm{DR})$; is obtained as

$$
S(\mathrm{DR})=\sum_{S L \pi} \sum_{\alpha} \frac{1}{2}(2 S+1)(2 L+1) P_{\alpha}^{S L \pi}
$$

The $\Omega_{\mathrm{DR}}$ are calculated, in a self-consistent manner, using the same CC wavefunction expansion that is used for the calculation of $\sigma_{\mathrm{PI}}$ : The DR cross section, in Megabarns (Mb), is related to the collision strength, $\Omega_{\mathrm{DR}}$; as

$$
\sigma_{\mathrm{DR}}(i \rightarrow j)(\mathrm{Mb})=\pi \Omega_{\mathrm{DR}}(i, j) /\left(g_{i} k_{i}^{2}\right)\left(a_{o}^{2} \times 10^{18}\right)
$$

where $k_{i}^{2}$ is the incident electron energy in Ry.

\subsection{Radiation damping of low-n resonances}

The dominant DR contribution from the energy region below series limits due to radiation damping of high- $n$ resonances of group (ii), with $n_{o}<n \leq \infty$; is considered using the BS theory as described above. Generally, for low- $n$ resonances of group (i) we do not expect radiation damping to be an important consideration since the autoionization rates are much larger than radiative decay rates. However, in some cases the effect could be significant and the low- $n$ resonance structures in the detailed photoionization cross sections need to be resolved and damped.

Although radiation damping of resonances in electron-ion interactions has been studied for a long time, the effect has been found to be important only for highly charged $\mathrm{H}$ - and He-like ions where the $2 p \rightarrow 1 s$ and the $1 s 2 p{ }^{1} P^{o} \rightarrow 1 s{ }^{21} S$ dipole transitions, respectively, correspond to radiative decay probabilities $\Gamma^{\mathrm{r}}$; of the order of $10^{13}-10^{14} \mathrm{~s}^{-1}$; approaching typical autoionization rates $\Gamma^{\text {a }}$. Employing the branching ratio between autoionization and radiative decay channels 
(Bates and Massey, 1943; Burgess, 1965; Presnyakov and Urnov, 1975), Pradhan (1981) showed DR may result in a significant reduction in the autoionization contribution to electron-ion scattering cross section. It was further pointed out that the flux lost from electron impact excitation (EIE) is equal to the DR contribution; detailed calculations were presented for He-like O VII and Fe XXV. Calculations for rate coefficients however showed that the net effect was not large. In a combined study of fine structure, autoionization, and radiative decays of high- $n$ resonances up to the Rydberg series limits at target thresholds, Pradhan (1983a, b) found maximum reductions in the EIE rate coefficients for the metastable $1{ }^{1} S_{0}-2{ }^{3} S_{1}$ transition of $9 \%$ for He-like Fe XXV and $19 \%$ for Mo XLI. The effect on EIE of the radiative decay of $l o w-n$ resonances is small. Following Pradhan's work, Trefftz (1983) studied several ionic systems and found that 'this effect is important only in certain cases of which highly charged He-like ions are the most prominent'.

Radiative decay of low- $n$ resonances in photoionization cross sections, relevant to $(e+$ ion $)$ recombination, is studied using two methods. The first, due to Sakimoto et al. (1990), entails fitting the dipole matrix element to a form

$$
D(E)=D^{0}(E)+\frac{A}{E-Z^{*}}, \quad Z=E_{0}-\frac{\mathrm{i}}{2} \Gamma^{\mathrm{a}},
$$

where $E_{0}$ is the resonance position and $\Gamma^{\mathrm{a}}$ is the autoionization width in Ry. The radiative decay width $\Gamma^{\mathrm{r}}$ is then obtained by

$\Gamma^{\mathrm{r}}=4 \pi^{2}|A|^{2} / \Gamma^{\mathrm{a}}$

The second-order radiative effects can then be included by considering

$$
D(E) \rightarrow \frac{D(E)}{1+L(E)}
$$

where, according to the BS theory, the operator L(E) is given by

$$
\begin{aligned}
L(E)= & \pi^{2}\left|D^{0}(E)\right|^{2}+2 \pi^{2} \frac{A^{*} D^{0}(E)}{E-Z} \\
& +2 \pi^{2} \frac{|A|^{2}}{(E-Z)\left(Z-Z^{*}\right)}
\end{aligned}
$$

A version of the BP R-matrix (BPRM) codes has been employed (Eissner W, to be submitted to Comput. Phys. Commun.); $\Gamma^{\mathrm{r}}$ are obtained in intermediate coupling from the BPRM calculations. Resonances and radiation damping in photoionization and photo-recombination may also be considered in the relativistic distorted-wave (RDW) approximation (Sampson and Zhang, 1995) accurate for highly charged ions. Using oscillator strengths or the radiation rates calculated by the Dirac-Fock-Slater (DFS) structure program (Sampson et al., 1989), and autoionization rates by the RDW program, the photo-excitation cross section from a level $k$ to a doubly excited level $j$ is given by the absorption radiative rate $A_{k j}^{\mathrm{r}}=h \Gamma_{k j}^{r}$

$$
\sigma_{k j}=\frac{4 \pi^{2} a_{0}^{2}}{\alpha^{2} E^{2}} \Gamma_{k j}^{\mathrm{r}} \delta\left(E-E_{j k}\right)
$$

where $E_{j k}$ is the transition energy, $a_{0}$ is the Bohr radius and $\alpha$ is the fine-structure constant. 
Replacing the $\delta$ function by a Lorentz profile, and from detailed balance with $g_{j} \Gamma^{\mathrm{r}}=g_{j} \Gamma_{\mathrm{j} \mathrm{k}}^{r}=$ $g_{k} \Gamma_{k j, j}^{r}$ obtain the photo-recombination cross section for the transition from levels $i$ to $k$ without radiation damping

$$
\sigma_{i k}^{\mathrm{PR}}=\sum_{j} \frac{\pi^{2} a_{0}^{2}}{E} \frac{g_{j}}{g_{i}} \Gamma^{\mathrm{T}} \frac{\Gamma^{\mathrm{a}} / 2 \pi}{\left(E-E_{j k}\right)^{2}+\left(\Gamma^{\mathrm{a}} / 2\right)^{2}},
$$

where $g$ is the statistical weight. When radiation damping is included the autoionization width $\Gamma^{\mathrm{a}}$ should be replaced in the denominator by the total width $\Gamma^{\mathrm{a}}+\Gamma^{\mathrm{r}}$ in the Lorentz profile, and we have

$$
\begin{aligned}
\sigma_{i k}^{\mathrm{PR}}= & \sum_{j} \frac{\pi^{2} a_{0}^{2}}{E} \frac{g_{j}}{g_{i}} \Gamma^{\mathrm{a}} \frac{\Gamma^{\mathrm{T}}}{\Gamma^{\mathrm{a}}+\Gamma^{\mathrm{y}}} \\
& \times \frac{\left(\Gamma^{\mathrm{a}}+\Gamma^{\mathrm{T}}\right) / 2 \pi}{\left(E-E_{j k}\right)^{2}+\left(\Gamma^{\mathrm{a}} / 2+\Gamma^{\top} / 2\right)^{2}} .
\end{aligned}
$$

This is the usual equation for the di-electronic recombination cross section such as that used in the RDW calculations (Sampson and Zhang, 1995), $\Gamma^{\mathrm{r}} /\left(\Gamma^{\mathrm{a}}+\Gamma^{\mathrm{r}}\right)$ being the branching ratio.

In a detailed study, Pradhan and Zhang (1997) showed that the fitting procedure is accurate and also provides a check for the version of the BPRM codes used. They found approximately $10-20 \%$ agreement between calculated dielectronic-resonance strengths for the KLL group of resonances in recombination with Fe XXV, and the corresponding values derived experimentally by Beiersdorfer et al. (1992), and other calculations.

In general, our studies have shown that with the exception of ions with $\mathrm{H}$ - and He-like core transitions, radiative decay of low- $n$ resonances do not show an appreciable effect on effective recombination cross sections (except in isolated or small energy ranges) or rate coefficients (e.g. Zhang et al., 2001).

\subsection{High-n background recombination-“"top-up”'}

At very low electron energies and temperatures the recombination is dominated by radiative recombination to very high- $n$ states just below the ground state of the recombining ion. These contributions are derived as discussed in Nahar (1996b). They are included in the hydrogenic approximation. Although the recombination to high- $n$ states is dominated by DR at higher temperatures, the radiative part involving the photo-ionization cross section of high- $n$ states is "topped-up" with the hydrogenic recombination rate coefficient. For an ion with charge $z$, we have the $z$-scaled formula $\alpha_{R}(z, T)=\alpha_{R}\left(1, T / z^{2}\right)$, in terms of the recombination rate coefficient for neutral hydrogen. We calculate the hydrogenic recombination rate coefficients, $\alpha_{R}(z, T)$, for states $n=10-800$ employing photoionization cross sections of hydrogen obtained using the FORTRAN program by Storey and Hummer (1992), and for states $n=801$ to $\infty$ using the sum (Hummer, 1994)

$$
\Delta(n)=x_{n}\left(\frac{n}{n+1}\right)^{3}\left(\frac{1+n}{2}\right) .
$$

\section{Results and discussion}


We present a wide range of results for ions of varying complexity to demonstrate the generality of the self-consistent unified method, and physical interrelationships among complementary processes of photoionization, recombination, and excitation.

\subsection{Comparison with experiments}

As a test of the accuracy and resolution of the $(\mathrm{e}+\mathrm{ion})$ recombination cross sections and photoionization cross sections using the self-consistent approach based on the R-matrix method, we have carried out a number of detailed comparisons for both recombination and photoionization with available experiments discussed in the following section.

\subsubsection{Unified $(e+i o n)$ recombination cross sections}

Very high resolution experimental cross sections are now being measured for $(\mathrm{e}+\mathrm{ion})$ recombination on heavy ion synchrotron storage rings, the test storage ring (TSR) in Heidelberg, and CRYRING in Stockholm. Experimental verification of the unified $(\mathrm{e}+$ ion) recombination cross sections, with those measured in detail on ion storage rings, has been done for several (recombined) ions for which experimental data is available: C III (Mannervik et al., 1998; Schippers et al., 1999), C IV,C V,O VII (Zhang et al., 1999), Ar XIII (Zhang and Pradhan, 1997), Fe XVII (Savin et al., 1999).

Fig. 2 demonstrates recombination to Ne-like Fe XVII (Pradhan et al., 2001b; Zhang et al., 2001) with a BPRM calculation that includes only the first three levels, $2 s^{2} 2 p^{5}\left({ }^{2} P^{0}: 3 / 2,1 / 2\right)$, $2 s 2 p^{6}\left({ }^{2} S_{1 / 2}\right.$ levels), in the eigenfunction expansion of the recombining ion F-like Fe XVIII. The main DR contributions arise from the two

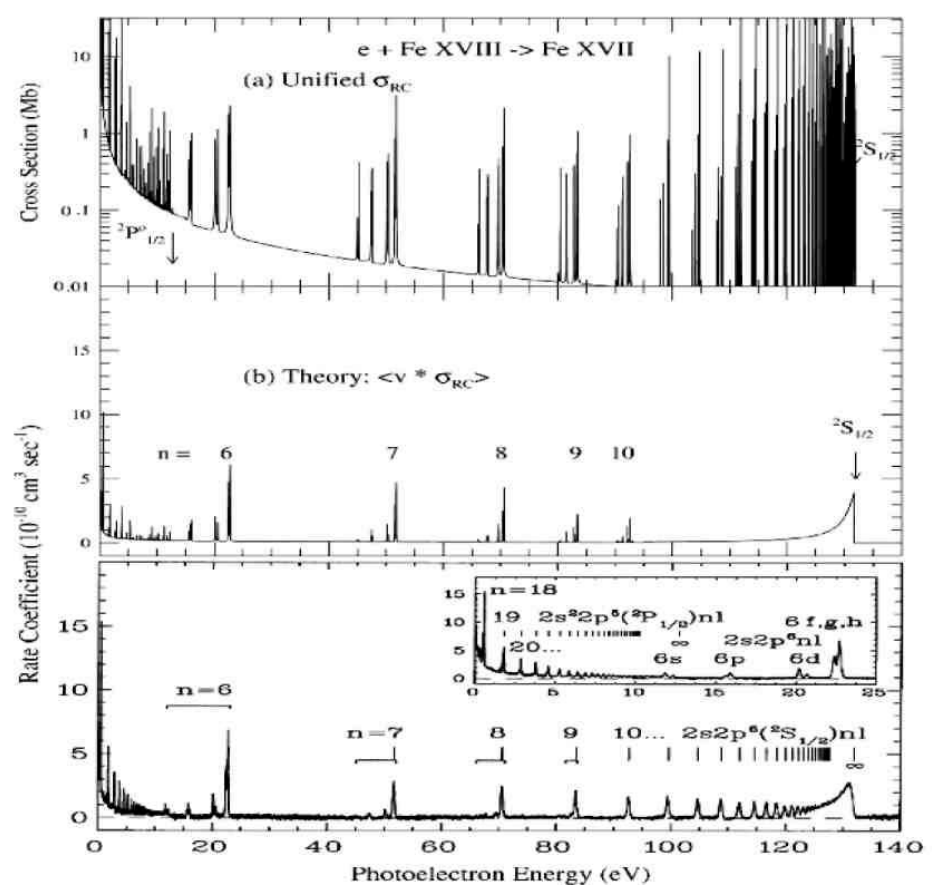

Fig. 2. Unified (e + FeXVIII) $\rightarrow$ FeXVII recombination cross sections (upper panel) with detailed resonance complexes below the $n=2$ thresholds of Fe XVIII (Pradhan et al., 2001b); Gaussian averaged over a 20 meV FWHM 
(middle panel); experimental data from ion storage ring measurements (bottom panel, Savin et al., 1999).
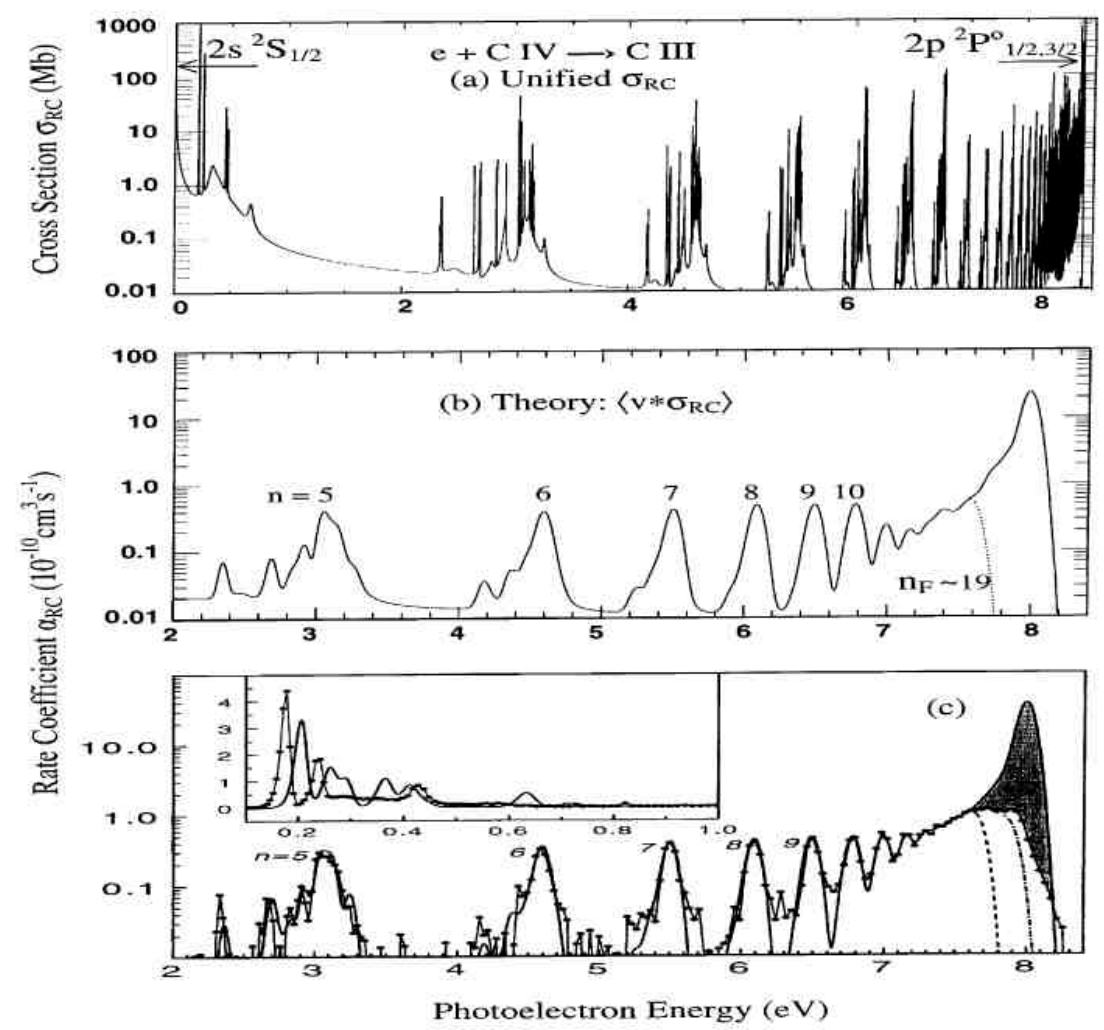

Fig. 3. (a) Unified (e + C IV) $\rightarrow$ C III recombination cross section $\sigma_{\mathrm{RC}}$ with detailed resonance structures (Pradhan et al., 2001b); (b) theoretical rate coefficient $\left(v \cdot \sigma_{\mathrm{RC}}\right)$ convolved over a Gaussian with experimental FWHM at the test storage ring (TSR) (Scippers et al., 2001); (c) the experimentally measured rate coefficient. The unified $\sigma_{\mathrm{RC}}$ in (a),(b) incorporate the background cross section eliminated from the experimental data in (c). The dashed and dot-dashed lines represent approximate field ionization cut-offs.

$\Delta n=0$ dipole transitions within the core ion. The computed rate coefficients agree with the sum of RR and experimentally derived DR rate coefficients (Savin et al., 1999) to within 20\%. A more extensive multi-configuration expansion up to the $n=3$ levels of Fe XVIII, with five spectroscopic configurations $2 s^{2} 2 p^{5}, 2 s 2 p^{6}, 2 s^{2} 2 p^{4}$ ( $3 s, 3 p, 3 d$ ), is in progress. These calculations will also include the $\Delta n=1$ dipole transitions that give rise to large resonances structures in the $n=2-3$ range, as found by Zhang et al. (2001).

Another set of relativistic close coupling BPRM calculations (Pradhan et al., 2001a, b) compared the unified $(\mathrm{e}+\mathrm{ion})$ recombination cross sections for recombination from Li-like C IV to Be-like C III with two different ion storage experiments, on CRYRING (Mannervik et al., 1998) and TSR (Scippers et al., 2001). Of particular interest in this case was the presence of large autoionizing $2 p 4 \ell$ resonances in the near-threshold region. The effective integrated value of the unified cross sections over this resonance complex lies between the two sets of experimental values, and agrees with both to about 15\%. Fig. 3 shows the detailed comparison of the BPRM results with the TSR data over the region covered by the experiment. Schippers et al. had earlier found that the previous $L S$ coupling calculations for carbon and nitrogen ions (Nahar and Pradhan, 1997) gave total C III rate coefficients that agreed with the sum of RR and experimental DR rate coefficients to within experimental uncertainties for all temperatures $T>5000 \mathrm{~K}$ : The discrepancy at lower temperatures was due to the $2 p 4 \ell$ complex, as discussed in detail by Mannervik et al. 
(1998) and Pradhan et al. (2001a), and resolved by the higher accuracy BPRM calculations shown in Fig. 3.

\subsubsection{Photoionization cross sections}

New advances have been made recently in measurements of photoionization cross sections with unprecedented resolution by three experimental groups: the University of Nevada, Reno, with the Advanced Light Source at Berkeley, Aarhus University, and University of Paris-Sud. All three experimental groups have

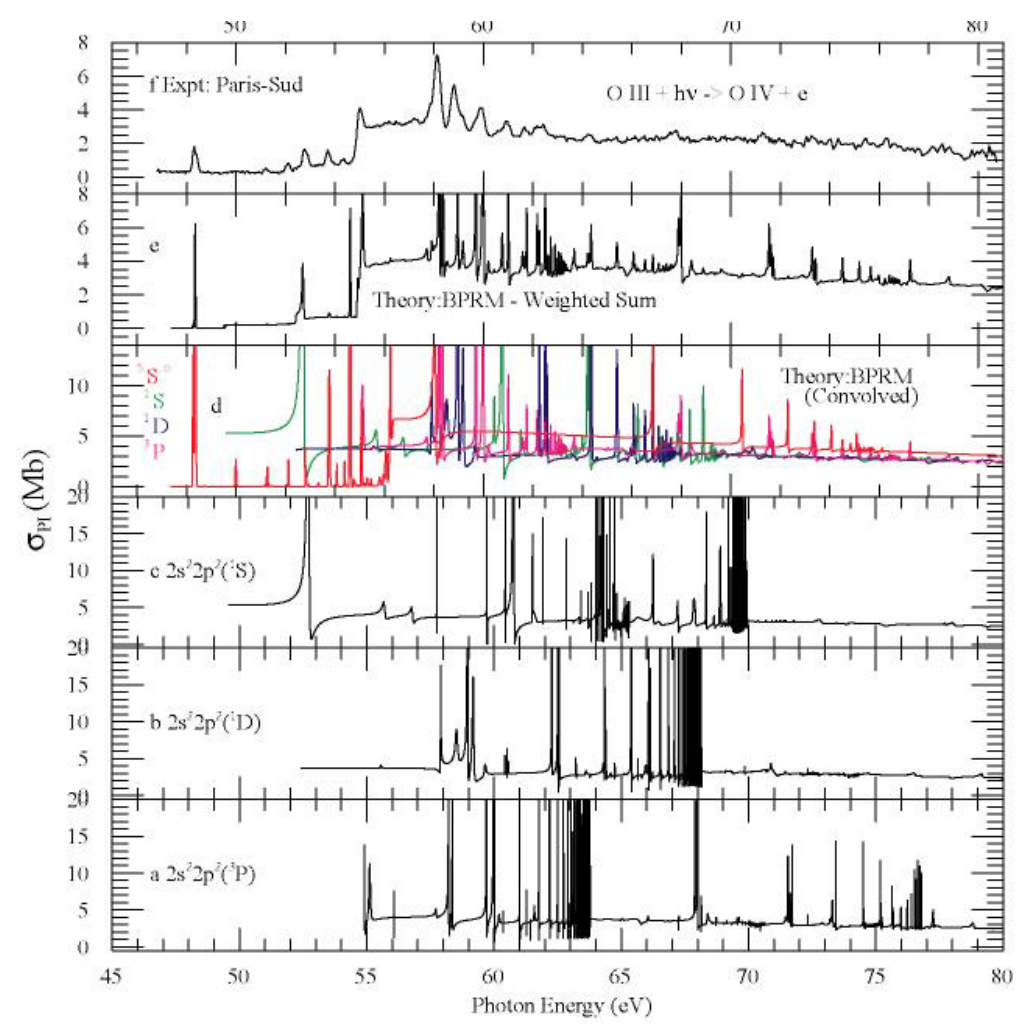

Fig. 4. Photoionization cross sections $\sigma_{\text {pr }}$ of O III (Nahar, 2004a): (a-c) $2 s^{2} 2 p^{2}\left({ }^{3} P,{ }^{1} D,{ }^{1} \mathrm{~S}\right.$ ) states, respectively; (d) convolved cross sections of ${ }^{3} \mathrm{P} ;{ }^{1} \mathrm{D} ;{ }^{1} \mathrm{~S} ;{ }^{5} \mathrm{~S}^{0}$. states over the experimental beam distribution; (e) weighted sum of convolved cross sections; (f) experimental cross sections measured at the University of Paris-Sud by Champeaux et al. (2004a).

compared their measured data with theoretical R-matrix calculations for several carbon and oxygen ions (e.g. Kjeldsen et al., 1999; Covingtion et al., 2001; Nahar, 2002, 2004a), as well as for heavier systems such as Fe II (Kjeldsen et al., 2002) and Fe IV (Gharaibeh et al., 2003). Fig. 4 shows a sample comparison between theory and experiment.

Of great interest is the comparison between theory and experiments to ascertain the mixture of ions in the ground and metastable levels in the experimental beam. The signature of metastable levels manifests itself in experimental data as resonances that appear below the ground state ionization threshold, since the metastable levels are photoionized at lower energies. Theoretical photoionization cross sections for the ground and metastable levels are therefore weighted and combined in order to compare with and interpret experimental measurements. This is likely to be of considerable importance in practical applications in laboratory and astrophysical 
plasmas where metastable levels may be significantly populated.

The level of sophistication of the state-of-the-art theoretical and experimental works on photoionization and $(\mathrm{e}+\mathrm{ion}$ ) recombination is evident from the examples in this section (and cited references). However, it needs to be emphasized that while theoretical calculations are capable of matching experimental accuracy and resolution, and vice versa, some physical effects and processes deserve careful consideration, as discussed later.

\subsection{Strongly coupled systems: iron ions}

The CC approximation is especially designed for strongly coupled systems. Among the most difficult R-matrix calculations are those for low ionization stages of iron. Hitherto, most of the work has been done in LS coupling since BPRM calculations are, as yet, intractable unless their scope is quite limited. In several previous calculations, the unified method has been applied to photoionization and recombination cross sections and rate coefficients for Fe $\mathrm{I}-\mathrm{V}$. The $\mathrm{CC}$
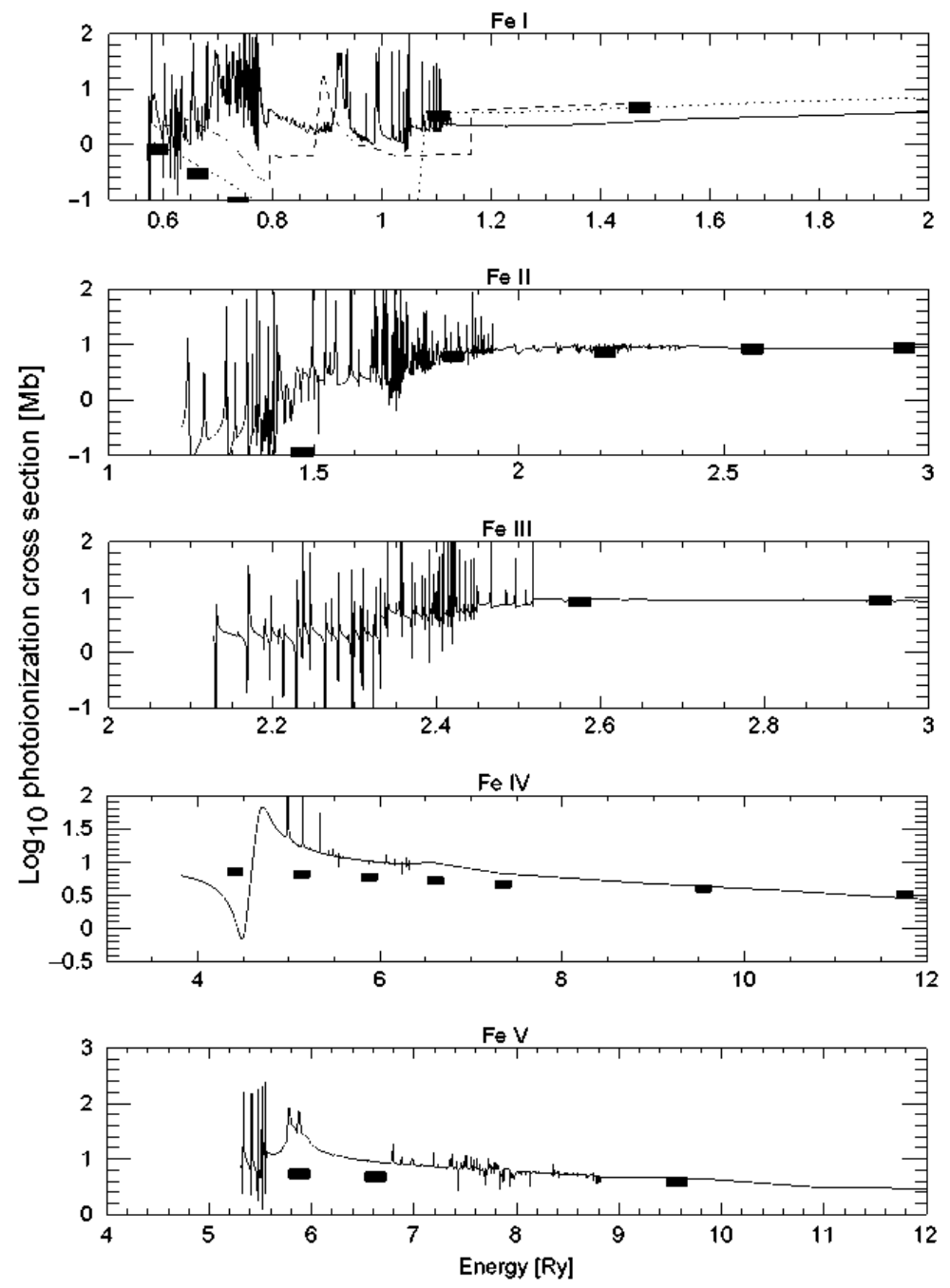

Fig. 5. Photoionization cross sections, $\sigma_{\text {PI }}$; of the ground states of Fe I-Fe V showing detailed autoionization resonances. Also plotted are the results of Reilman and Manson (1979, filled squares), Verner et al. (1993, dotted line), and Kelly (1972, dashed line in Fe I). 
expansions include all LS terms of the ground and excited even parity configuration of the recombining ion, and the first excited odd parity configuration that enables dipole transitions in the core.

The photoionization cross sections, $\sigma_{\mathrm{PI}}$; are calculated including autoionizing resonances that can enhance the background cross sections considerably. Fig. 5 shows the photoionization cross sections of the ground states of Fe I to Fe V (Bautista, 1996; Bautista and Pradhan, 1995, 1997; Nahar and Pradhan, 1994b; Nahar, 1996a). Extensive resonances dominate the cross sections for these complex ions. The differences with previous calculations, indicating resonance enhancement due to extensive channel couplings, are up to three orders of magnitude for Fe I, over an order or magnitude for Fe II, and $\sim 50 \%$ for Fe III. The primary reason for the differences with simpler approximations, such as the central field approximation, that neglect channel couplings, is due to the dominant role of the $3 d$-shell in photoionization of these Fe ions, relative to the much smaller cross section of the outer $n=4$ electrons with increasing energy. CC calculations are therefore essential to obtain accurate cross sections for these Fe-ions, and indeed for all neutral and low ionization states of elements. It is remarkable however that the earlier many-body perturbation theory calculations for Fe I by
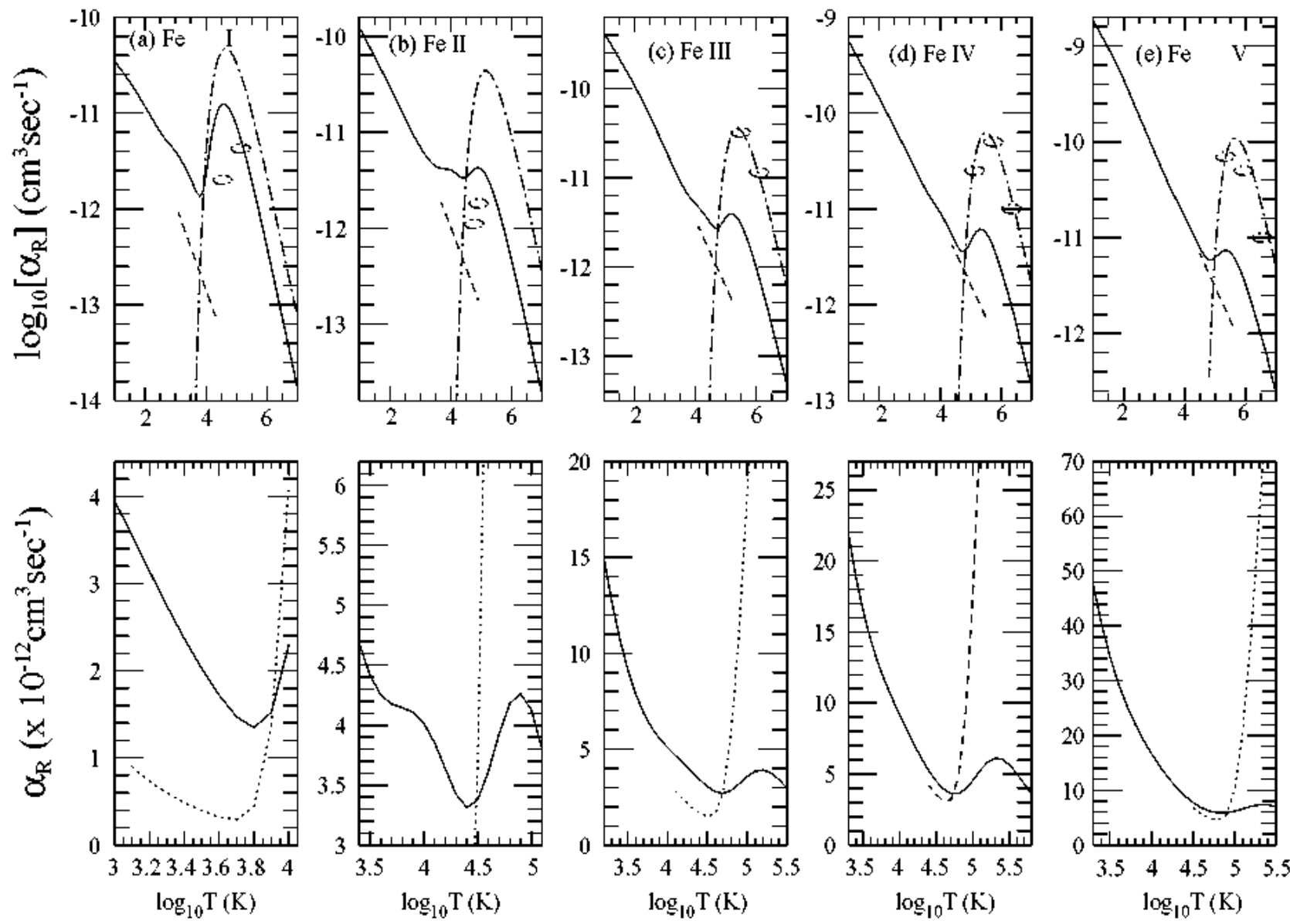

Fig. 6. Comparison of the unified total recombination rate coefficients $\left(\alpha_{R}(T)\right.$ (solid lines) for Fe I (Nahar et al., 1997), Fe II (Nahar, 1997), Fe III (Nahar, 1996a), Fe IV (Nahar et al., 1998), and Fe V (Nahar and Bautista, 1999). The unified rate coefficients are compared with the sum of RR and DR rate coefficients from Woods et al. (1981, dashed lines). 
Kelly (1972, dashed line in Fig. 5) show some similar structures as the R-matrix cross sections. The recombination rate coefficients for Fe I-V (Nahar, 1996a, 1997; Nahar and Bautista, 1999; Nahar et al., 1997, 1998) are shown in Fig. 6. These were computed using photoionization cross sections for the ground state (Fig. 5), and typically a several hundred of excited bound states. The CC expansion for the recombining ion included target states of configurations denoted as $3 d^{q}(4 s, 4 p, 4 d)$. For instance the target expansion for photoionization and recombination of Fe II: (e $+\mathrm{Fe}$ III $\leftrightarrow \mathrm{Fe}$ II $+h v$, employ an 83-LS term expansion for core ion Fe III with configurations $3 d^{6}, 3 d^{5}(4 s, 4 p, 4 d)$ (Nahar and Pradhan, 1994b). The low-energy resonance structures are well represented, as seen in Fig. 5, including the dipole $3 d \rightarrow 4 p$ transitions responsible for DR. Therefore, at low-temperatures $T<10^{5} \mathrm{~K}$ the unified $\alpha_{R}(T)$ should be quite accurate. We find considerable differences with previous works on RR and DR of Fe ions. For example, for Fe I the unified recombination rate is up to a factor of 4 higher at low temperatures than the sum of RR and DR rate coefficients from Woods et al. (1981).

Owing to the size of the computational problem, the much higher energy $3 d \rightarrow 4 f$ core transitions were not included in the CC expansion for the target ions. This likely accounts for the discrepancy in the higher temperature range from the DR bump in $\alpha_{R}(T)$ : In fact there are two competing effects: enhancement due to the $3 d \rightarrow 4 f \mathrm{DR}$, and reduction due to autoionization into many excited states present below the high-lying $3 d^{q} 4 f$ levels. More extensive calculations are needed to fully study this issue. Fine structure also needs to be considered in order to obtain higher accuracy low-energy cross sections and $\alpha_{\mathrm{R}}(T)$ :

Table 1 gives the unified $\alpha_{\mathrm{R}}(T)$ for Fe I-V in the low-temperature region where they are usually abundant in plasmas. Also given in Table 1 are sample $\alpha_{R}(T)$ from recent works for some other iron ions. The unified method, based on the CC approximation, is possibly the only method capable of accurate results for neutral and low ionization states of elements, where a separate treatment of RR and DR is unphysical and inaccurate. The R-matrix method for ( + ion) recombination is of course equally valid and accurate for highly charged ions where a separation between the background and resonances is easier, and the sum of individual RR and DR rate coefficients may be of sufficient accuracy. Table 1 also gives the total unified $\alpha_{R}(T)$ for a few highly ionized iron ions (Nahar, 2000; Nahar, 2002) displaying the full range of ionization states from Fe I to Fe XXV.

\subsection{DR collision strengths}

Recombination into high- $n$ bound levels of the $(e+$ ion) system is treated analytically using the $\mathrm{CC}$ formulation that is an extension of electron impact excitation to radiation damping of resonances and DR (BS 1985, Nahar and Pradhan, 1994a), as described in the theory section.

Fig. 7 shows a typical DR collision strength with high $n\left(n>n_{o} \approx 10\right)$ resonances. These calculations employ the same wavefunction expansion as the detailed photoionization/ photorecombination calculations for all possible low- $n$ (SLJ) levels with $n \leq n_{0}$ :

\subsection{Photoexcitation-of-core resonances and DR}

One of the main physical features of the present approach, self-consistent treatment of photoionization 
Table 1

Total recombination rate coefficients, $\alpha_{R}(T)$ (in units of $\mathrm{cm}^{3} \cdot \mathrm{s}^{-1}$ ) for iron ions in the temperature range of $1.0 \leq$ $\log _{10}(T) \leq 9.0$

\begin{tabular}{|c|c|c|c|c|c|c|c|c|c|}
\hline \multirow[t]{2}{*}{$\log _{10} T$} & \multicolumn{9}{|l|}{$\alpha_{\mathrm{R}}(T)$} \\
\hline & Fe I & $\mathrm{Fe}$ II & Fe III & Fe IV & $\mathrm{Fe} \mathrm{V}$ & Fe XIII & $\mathrm{Fe} X \mathrm{XIV}$ & Fe XXV & Fe XXVI \\
\hline 1.0 & $3.48-11$ & 1.23 .10 & $4.20-10$ & $5.57 \cdot 10$ & $1.80-9$ & $1.84-8$ & $2.38-08$ & $2.80-8$ & $3.21-8$ \\
\hline 1.1 & $3.14-11$ & $1.07-10$ & $3.67-10$ & $4.88-10$ & $1.58-9$ & $1.63-8$ & $2.11-08$ & $2.49-8$ & $2.85-8$ \\
\hline 1.2 & $2.84-11$ & 9.3411 & $3.22-10$ & $4.28-10$ & $1.39-9$ & $1.44-8$ & $1.88-08$ & $2.21-8$ & 2.548 \\
\hline 1.3 & $2.58-11$ & $8.13-11$ & $2.82-10$ & $3.74-10$ & 1.22 .9 & $1.27-8$ & $1.67-08$ & 1.97 .8 & 2.25 .8 \\
\hline 1.4 & $2.33-11$ & $7.06-11$ & $2.47-10$ & $3.27 \cdot 10$ & 1.07 .9 & $1.12-8$ & $1.48-08$ & $1.75 \cdot 8$ & $2.00-8$ \\
\hline 1.5 & $2.09-11$ & $6.13-11$ & $2.16-10$ & $2.86-10$ & 9.3410 & $9.81-9$ & $1.31-08$ & $1.55-8$ & $1,78-8$ \\
\hline 1.6 & $1.87-11$ & $5.32-11$ & $1.89-10$ & $2.49-10$ & $8.12-10$ & $8.59-9$ & $1.16-08$ & $1.38-8$ & $1.58-8$ \\
\hline 1.7 & $1.67-11$ & $4.61-11$ & $1.65-10$ & $2.17 \cdot 10$ & $7.03-10$ & $7.50-9$ & $1.03-08$ & 1.22 .8 & $1.40-8$ \\
\hline 1.8 & $1.48-11$ & $3.98-11$ & $1.44-10$ & $1.89 \cdot 10$ & $6.05 \cdot 10$ & $6.53-9$ & $9.13-09$ & $1.08-8$ & $1.24-8$ \\
\hline 1.9 & $1.31-11$ & $3.45-11$ & $1.25-10$ & $1.65-10$ & $5.18-10$ & $5.67-9$ & $8.06-09$ & $9.55-9$ & $1.10-8$ \\
\hline 2.0 & $1.16-11$ & $2.97-11$ & $1.08-10$ & $1.43-10$ & $4.40-10$ & $4.89-9$ & $7.12-09$ & $8.44-9$ & $9.71-9$ \\
\hline 2.1 & $1.02-11$ & $2.56-11$ & $9.30-11$ & $1.24-10$ & $3.72 \cdot 10$ & $4.21-9$ & $6.28-09$ & $7.46 \cdot 9$ & 8.58 .9 \\
\hline 2.2 & $9.02-12$ & $2.21 \cdot 11$ & $7.99-11$ & $1.08-10$ & $3.13-10$ & $3.60-9$ & $5.53-09$ & $6.58-9$ & 7.58 .9 \\
\hline 2.3 & $7.97-12$ & $1.90-11$ & $6.84-11$ & $9.38-11$ & $2.63-10$ & $3.07-9$ & $4.87-09$ & $5.80-9$ & $6.69-9$ \\
\hline 2.4 & $7.04-12$ & $1.63-11$ & $5.83-11$ & $8.13-11$ & $2.21-10$ & $2.63-9$ & $4.29-09$ & $5.11-9$ & $5.90-9$ \\
\hline 2.5 & $6.27-12$ & $1.40-11$ & $4.95-11$ & $7.06 \cdot 11$ & $1.86-10$ & $2.26-9$ & $3.76-09$ & 4.50 .9 & $5.20-9$ \\
\hline 2.6 & $5.65-12$ & $1.21-11$ & $4.19-11$ & $6.11 \cdot 11$ & $1.57 \cdot 10$ & $1.97-9$ & $3.30-09$ & $3.95-9$ & 4.57 .9 \\
\hline 2.7 & $5.15-12$ & $1.04-11$ & $3.54-11$ & $5.29-11$ & $1.32-10$ & $1.77-9$ & $2.90-09$ & $3,47-9$ & $4.02-9$ \\
\hline 2.8 & $4.73-12$ & $9.03-12$ & $2.98-11$ & $4.57-11$ & $1.12-10$ & $1.61-9$ & $2.54-09$ & $3.05-9$ & $3.53-9$ \\
\hline 2.9 & $4.34-12$ & 7.91 .12 & $2.50-11$ & $3.95-11$ & $9.40-11$ & $1.49-9$ & $2.22-09$ & 2.67 .9 & $3.10-9$ \\
\hline 3.0 & $3.95-12$ & $6.97 \cdot 12$ & $2.10-11$ & $3.41 \cdot 11$ & $7.92-11$ & $1.38-9$ & $1.94-09$ & $2.34-9$ & 2.72 .9 \\
\hline 3.1 & $3.55-12$ & $6.20-12$ & $1.77-11$ & 2.93-11 & $6.67-11$ & $1.28-9$ & $1.70-09$ & $2.05-9$ & $2.39-9$ \\
\hline 3.2 & $3.14-12$ & $5.57-12$ & $1.49-11$ & $2.53-11$ & $5.62-11$ & $1.19-9$ & $1.48-09$ & $1.79-9$ & $2.09-9$ \\
\hline 3.3 & $2.75-12$ & $5.07 \cdot 12$ & $1.26-11$ & $2.18-11$ & $4.75-11$ & $1.12-9$ & $1.29-09$ & 1.57 .9 & 1.83 .9 \\
\hline 3.4 & $2.37-12$ & $4.68 \cdot 12$ & $1.07-11$ & $1.88-11$ & $4.03-11$ & $1.08-9$ & $1.13-09$ & 1.37 .9 & $1.60-9$ \\
\hline 3.5 & $2.02-12$ & $4.41-12$ & $9.14-12$ & $1.64-11$ & $3,44-11$ & $1.06-9$ & $9.81-10$ & $1.20-9$ & $1.40-9$ \\
\hline 3.6 & $1.72-12$ & $4.25-12$ & $7.86-12$ & $1.45-11$ & $2.95-11$ & $1.06-9$ & $8.54-10$ & $1.04-9$ & $1.23-9$ \\
\hline 3.7 & $1.47-12$ & $4.18-12$ & $6.86-12$ & $1.28-11$ & $2.56-11$ & $1.06-9$ & $7.42-10$ & $9.11-10$ & $1.07-9$ \\
\hline 3.8 & $1.35-12$ & $4.14-12$ & $6.11-12$ & $1.15-11$ & $2.22-11$ & $1.05-9$ & $6.45-10$ & 7.94-10 & $9.35 \cdot 10$ \\
\hline 3.9 & $1.52-12$ & $4.11-12$ & $5.54-12$ & $1.03-11$ & $1.93-11$ & $1.02-9$ & $5.60-10$ & $6.91-10$ & $8.16-10$ \\
\hline 4.0 & $2.30-12$ & $4,02-12$ & $5.08-12$ & $9.14-12$ & $1.67-11$ & $9.68-10$ & $4.86-10$ & $6.01-10$ & $7.11-10$ \\
\hline 4.1 & $3.89-12$ & $3.85-12$ & $4.66-12$ & $8.06-12$ & $1.44-11$ & $8.92-10$ & $4.22-10$ & $5.24-10$ & $6.21-10$ \\
\hline 4.2 & $6.19-12$ & $3.63-12$ & $4.26-12$ & $7.03 \cdot 12$ & $1.23-11$ & $8.00-10$ & $3.65-10$ & $4.55 \cdot 10$ & $5.41 \cdot 10$ \\
\hline 4.3 & $8.72-12$ & $3,42-12$ & $3.84-12$ & $6.06-12$ & $1.05-11$ & $7.02-10$ & $3.16-10$ & $3.96-10$ & $4.70-10$ \\
\hline 4.4 & $1.09-11$ & $3.31-12$ & $3.43-12$ & $5.19-12$ & $8.99-12$ & $6.07-10$ & $2.73-10$ & $3.44-10$ & $4.10-10$ \\
\hline 4.5 & $1.21-11$ & $3.38-12$ & $3.05-12$ & $4.48-12$ & $7.75-12$ & $5.22-10$ & $2.36-10$ & $2.98-10$ & $3.56-10$ \\
\hline 4.6 & $1.24-11$ & $3.61 \cdot 12$ & $2.78-12$ & 3.93 .12 & $6.81 \cdot 12$ & $4.49-10$ & $2.04-10$ & $2.59 \cdot 10$ & $3.10-10$ \\
\hline 4.7 & $1.17-11$ & $3.93-12$ & $2.70-12$ & $3.63-12$ & $6.19 \cdot 12$ & $3.87-10$ & $1.76-10$ & $2.24-10$ & $2.69-10$ \\
\hline 4.8 & $1.05-11$ & $4.18-12$ & $2.85-12$ & $3.62-12$ & $5.88-12$ & $3.37-10$ & $1.52-10$ & $1.94-10$ & $2.34-10$ \\
\hline 4.9 & $8.95-12$ & $4.26-12$ & $3.19-12$ & $3,95-12$ & $5.89-12$ & $2.94-10$ & $1.31-10$ & $1.68-10$ & $2.03-10$ \\
\hline 5.0 & $7.35-12$ & 4.13 .12 & $3.58-12$ & $4.56 \cdot 12$ & $6.18 \cdot 12$ & $2.59-10$ & $1.12-10$ & $1.45 \cdot 10$ & $1.76 \cdot 10$ \\
\hline 5.1 & - & - & - & - & - & $2.30-10$ & $9.67-11$ & $1.25 \cdot 10$ & $1.52 \cdot 10$ \\
\hline 5.2 & - & - & - & - & - & $2.06-10$ & $8.30-11$ & $1.08-10$ & $1.32-10$ \\
\hline 5.3 & - & - & - & - & - & $1.84-10$ & $7.12-11$ & $9.32-11$ & $1.14-10$ \\
\hline 5.4 & - & - & - & - & - & $1.64-10$ & $6.10-11$ & $8.03-11$ & $9.85-11$ \\
\hline 5.5 & - & - & - & - & - & $1.43-10$ & $5.22-11$ & $6.91 \cdot 11$ & $8.51-11$ \\
\hline 5.6 & - & - & - & - & - & $1.23-10$ & $4.47-11$ & $5.94-11$ & $7.34-11$ \\
\hline 5.7 & - & - & - & - & - & $1.03-10$ & $3.81-11$ & $5.10-11$ & $6.33-11$ \\
\hline 5.8 & - & - & - & - & - & $8.39-11$ & $3.25-11$ & $4.37-11$ & $5.45-11$ \\
\hline 5.9 & - & - & - & - & - & $6.72-11$ & $2.77-11$ & $3.74-11$ & $4.69-11$ \\
\hline 6.0 & - & - & - & - & - & $5.28-11$ & $2.35-11$ & $3,20-11$ & $4.02-11$ \\
\hline 6.1 & - & - & - & - & - & $4.09-11$ & $1.99-11$ & 2.73-11 & $3.46-11$ \\
\hline 6.2 & - & - & - & - & - & $3.12-11$ & $1.69-11$ & $2.32-11$ & $2.96-11$ \\
\hline 6.3 & - & - & - & - & - & $2.36-11$ & $1.42-11$ & $1.98 \cdot 11$ & $2.53-11$ \\
\hline 6.4 & - & - & - & - & - & $1.77-11$ & $1.20-11$ & $1.68-11$ & $2.17-11$ \\
\hline
\end{tabular}


Table 1 (continued)

\begin{tabular}{|c|c|c|c|c|c|c|c|c|c|}
\hline \multirow[t]{2}{*}{$\log _{10} T$} & \multicolumn{9}{|c|}{$\alpha_{\mathrm{R}}(T)$} \\
\hline & Fe I & $\mathrm{Fe}$ II & $\mathrm{Fe}$ III & Fe IV & $\mathrm{Fe} \mathrm{V}$ & Fe XIII & Fe XXIV & Fe XXV & Fe XXVI \\
\hline 6.5 & - & - & - & - & - & $1.32-11$ & $1.00-11$ & $1.42-11$ & $1.85-11$ \\
\hline 6.6 & - & - & - & - & - & $9.80-12$ & $8.40-12$ & $1.20-11$ & $1.58-11$ \\
\hline 6.7 & - & - & - & - & - & $7.23-12$ & $7.00-12$ & $1.02-11$ & $1.34-11$ \\
\hline 6.8 & - & - & - & - & - & $5.34-12$ & $5.82-12$ & $8.56-12$ & $1.14-11$ \\
\hline 6.9 & - & - & - & - & - & $3.94-12$ & $4.83-12$ & $7.20-12$ & $9.64-12$ \\
\hline 7.0 & - & - & - & - & - & $2.90-12$ & $4.03-12$ & $6.06-12$ & $8.14-12$ \\
\hline 7.1 & - & - & - & - & - & $2.04-12$ & $3.41-12$ & $5.13-12$ & $6.88-12$ \\
\hline 7.2 & - & - & - & - & - & $1.50-12$ & $2.98-12$ & $4.38-12$ & $5.78 \cdot 12$ \\
\hline 7.3 & - & - & - & - & - & $1.09-12$ & $2.70-12$ & $3.79 \cdot 12$ & $4.85-12$ \\
\hline 7.4 & - & - & - & - & - & $7.93-13$ & $2.51-12$ & $3,31-12$ & $4.06-12$ \\
\hline 7.5 & - & - & - & - & - & $5.79-13$ & $2.35-12$ & $2.89-12$ & $3.38-12$ \\
\hline 7.6 & - & - & - & - & - & $4.23-13$ & $2.16-12$ & $2.52-12$ & $2.81 \cdot 12$ \\
\hline 7.7 & - & - & - & - & - & $3.08-13$ & $1.94-12$ & $2.17 \cdot 12$ & $2.32-12$ \\
\hline 7.8 & - & - & - & - & - & $2.25-13$ & $1.70-12$ & $1.84-12$ & $1.92-12$ \\
\hline 7.9 & - & - & - & - & - & $1.65-13$ & $1.43-12$ & $1.53-12$ & $1.57-12$ \\
\hline 8.0 & - & - & - & - & - & $1.20-13$ & $1.18-12$ & $1.25 \cdot 12$ & $1.28 \cdot 12$ \\
\hline 8.1 & - & - & - & - & - & $8.81-14$ & $9.49-13$ & $1.01-12$ & $1.04-12$ \\
\hline 8.2 & - & - & - & - & - & $6.45-14$ & $7.47-13$ & $8.07 \cdot 13$ & $8.40-13$ \\
\hline 8.3 & - & - & - & - & - & $4.74-14$ & $5.79-13$ & $6.36-13$ & $6.73-13$ \\
\hline 8.4 & - & - & - & - & - & $3.48-14$ & $4.42-13$ & $4.97-13$ & 5.37 .13 \\
\hline 8.5 & - & - & - & - & - & $2.57-14$ & $3.34-13$ & $3.85-13$ & $4.26-13$ \\
\hline 8.6 & - & - & - & - & - & $1.90-14$ & $2.51-13$ & $2.97 \cdot 13$ & $3.36-13$ \\
\hline 8.7 & - & - & - & - & - & $1.41-14$ & $1.86-13$ & $2.27-13$ & $2.63-13$ \\
\hline 8.8 & - & - & - & - & - & $1.05-14$ & $1.38-13$ & $1.73 \cdot 13$ & $2.05-13$ \\
\hline 8.9 & - & - & - & - & - & $7.86-15$ & $1.02-13$ & $1.32-13$ & $1.59-13$ \\
\hline 9.0 & - & - & - & - & - & $5.92-15$ & $7.46-14$ & $9.94-14$ & $1.22-13$ \\
\hline
\end{tabular}

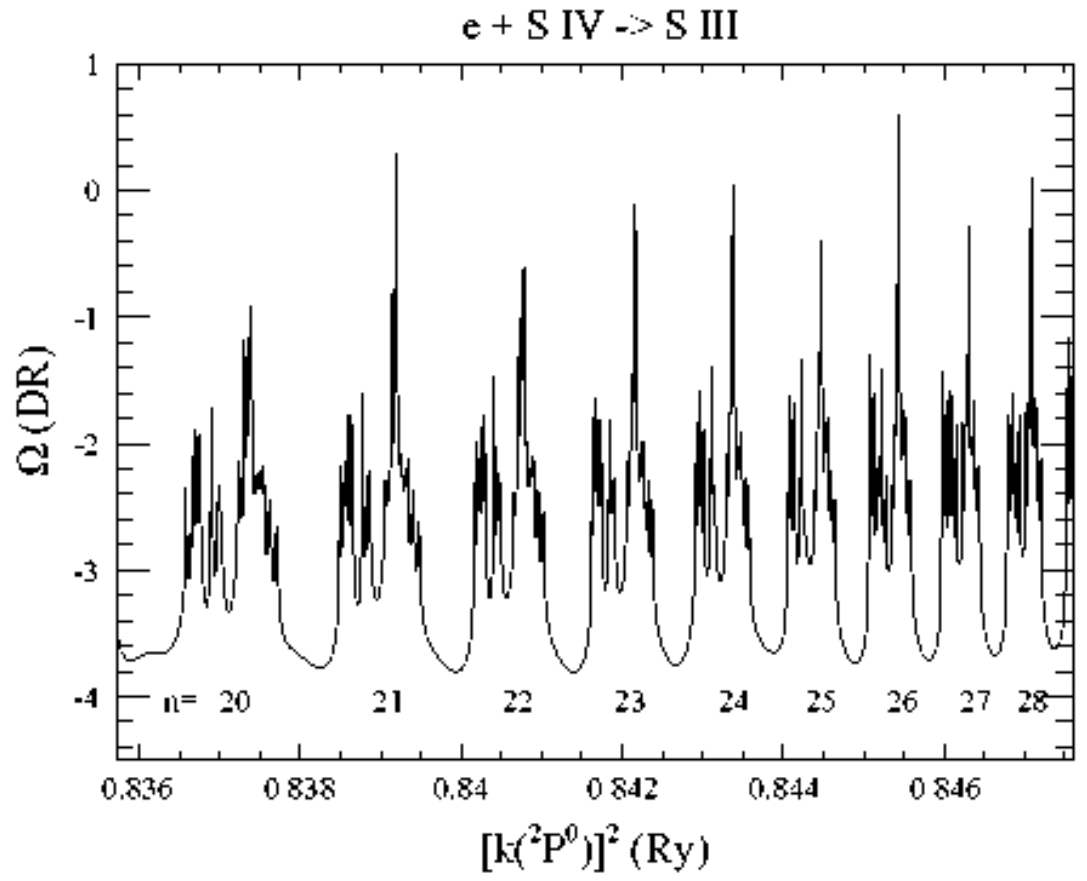

Fig. 7. DR collision strengths for high- $n(10<n \leq \infty)$ resonances in $(\mathrm{e}+\mathrm{SIV}) \rightarrow \mathrm{S}$ III recombination (Nahar and Pradhan, 1994a). 


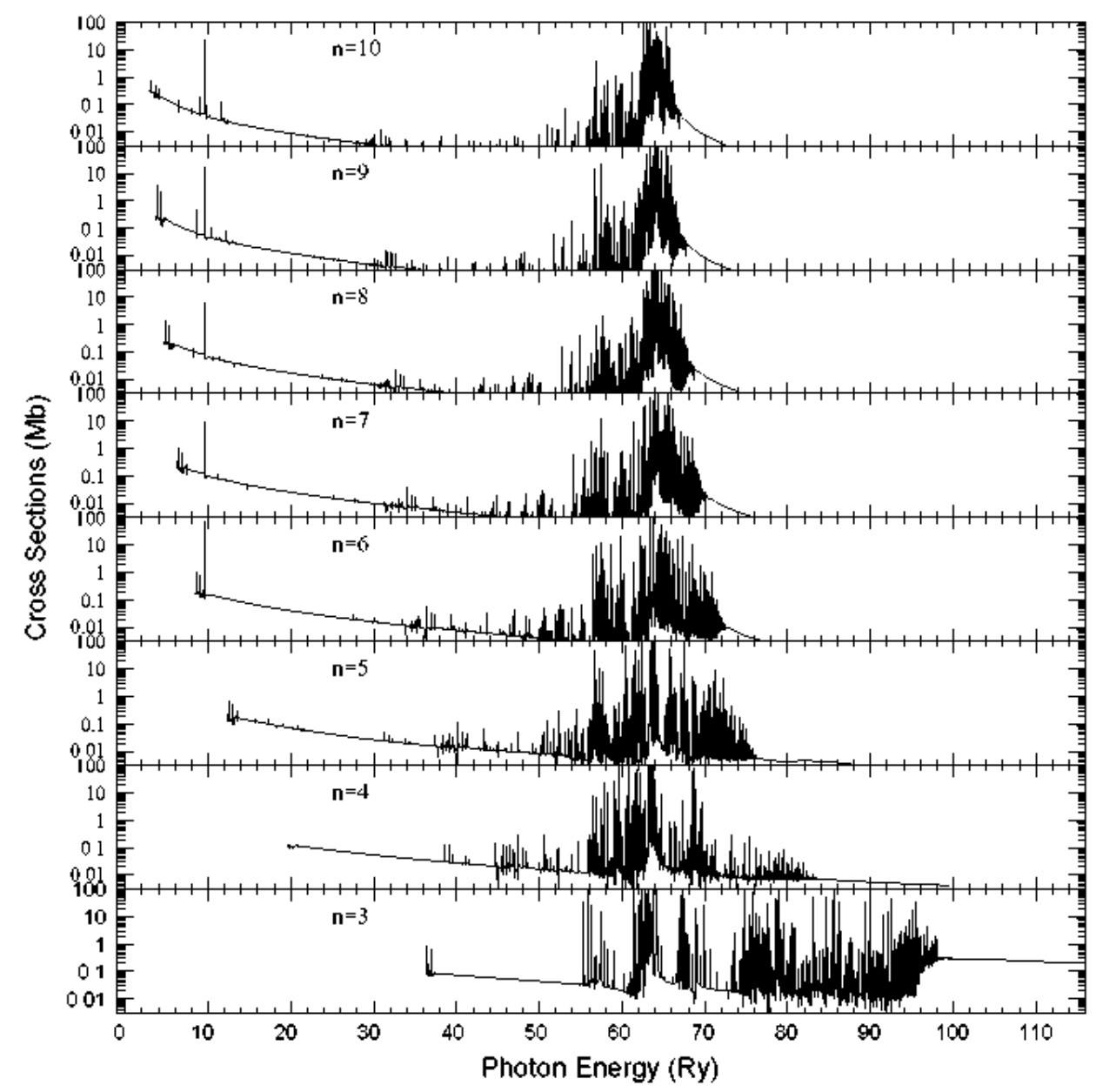

Fig. 8. Photoexcitation-of-core (PEC) resonances in photoionization of the $2 p^{5} n p\left({ }^{3} P_{0}\right)$ Rydberg series of levels of Fe XVII. The giant PEC resonance feature at approximately 63 Ry corresponds to strong dipole excitations in the transition arrays $2 p^{5}-2 p^{4}$. (3s, 3d) within the Fe XVIII core (60 CC results, Zhang et al., 2001).

and recombination, is exemplified by the relationship between photoexcitation-of-core (PEC) resonances and the inverse process of DR. Photoionization of bound levels along a Rybderg series exhibit large PEC resonances at series limits corresponding to dipole transitions in the core ion (Yuand Seaton, 1987; Nahar and Pradhan, 1992). The PECs generally attenuate the cross section by orders of magnitude relative to the background.

Fig. 8 illustrates the PEC resonances in the photoionization of a Rydberg series of excited bound levels of Fe XVII. The first noteworthy point is that the huge rise in the cross section occurs in what is otherwise expected to be hydrogenic behavior, since the photoionized levels belong to a Rydberg series for a given $n S L \pi$ or $n J \pi$ symmetry of the (e + ion) system; the higher the $n$ value the more outstanding the PEC feature. This also points to the fact that photoionization of excited levels of a non-hydrogenic ion may not be treated in hydrogenic approximation, as is often done in practical plasma applications.

The inverse of PEC is DR. Electrons that excite the core ion may be captured into high- $n$ autoionizing resonances, which subsequently decay radiatively to corresponding high- $n$ bound levels of the $(e+i o n)$ system. Such excitations generally involve strong dipole transitions in the core and PEC resonances occur at photon energies equal to these transitions. 


\section{Physical effects and processes}

\subsection{The unified approach and separate RR and DR treatments}

The unified (e + ion) recombination rate coefficients $\alpha_{R}(T)$ are valid over a wide range of temperatures for all practical purposes. In contrast, separate calculation of RR and DR rate coefficients are carried out in different approximations valid for limited temperature ranges, such as low-temperature DR, high-temperature DR, and RR. Moreover, division is sometimes made between $\Delta n=0$ and $\Delta n \neq 0$ transitions in DR (Savin et al., 1999). The unified cross sections generally cover all temperature ranges, with cross sections computed over extended energy ranges.

However, the main problem with separate treatment of RR and DR is more fundamental. Even if the DR treatment is satisfactory, the calculation of RR rate coefficient would require the calculation of unphysical photoionization cross sections without resonances, computed in simpler approximations, such as the central field method that does not include resonances, or a "one-channel”' R-matrix calculation (Gorczyca et al., 2002) that contradicts the CC approach which, by definition, incorporates the necessary physics of channel coupling as manifested in resonances. A self-consistent and physical treatment to $(\mathrm{e}+\mathrm{ion})$ recombination is enabled by the use of CC wavefunctions employed in the unified approach.

\subsection{Correspondence between photorecombination, dielectronic recombination, and excitation}

The present close-coupling treatment of (electron-ion) recombination is an unified and integrated approach to photorecombination (PR), DR, and electron impact excitation (EIE). Fig. 9 illustrates the inter-relationships required by conservation-of-flux and unitarity conditions for PR, $\mathrm{DR}$, and EIE for the (e + C VI) $\rightarrow$ C V system (Zhang et al., 1999). The cross sections for all three processes, computed independently but with the same $\mathrm{CC}$ wavefunction expansion, are continuous functions of energy. The PR cross sections include the background non-resonant contribution as well as the resonances (left of the dashed line in Fig. 9), whereas the DR cross sections (right of the dashed line), computed using the BS theory, neglect the background contribution. The two cross sections, the PR and DR, match smoothly at $v \approx 10.0$ showing that the background contribution is negligible compared to the resonant contribution at high $n>10$ : Further, the DR cross sections rise exactly up to the EIE cross section at the threshold of excitation according to the theoretical condition at threshold

$$
\lim _{n \rightarrow \infty} \Omega_{\mathrm{DR}}(n)=\lim _{k^{2} \rightarrow 0} \Omega_{\mathrm{EIE}}\left(k^{2}\right) .
$$

The DR cross section in Fig. 9 at the series limit $2{ }^{1} P_{1}$ agrees precisely with the independently determined value of the electron impact excitation cross section (filled circle) for the dipole transition $1{ }^{1} S_{0}-2{ }^{1} P_{1}$; as required by the unitarity condition for the generalized $S$-matrix and conservation of flux. The continuous transition between the PR, DR, and EIE cross sections serves to validate the accuracy of the BS theory of DR. The DR cross sections are, on the one hand, consistent with an extensively detailed coupled channel treatment of photorecombination, until an energy region where background recombination is insignificant, and, on the other hand, consistent with the threshold behaviour at the EIE threshold. In fact Eq. (27) provides a powerful accuracy check on the possible importance of long range multipole potentials, partial wave summation, level degeneracies at threshold, and other numerical inaccuracies 
(discussed in previous works).

The radiatively damped cross sections in Fig. 9 illustrate that, owing to the interaction with the radiation field, the autoionizing resonances are broadened, smeared, and wiped out (in that order) as $n \rightarrow \infty$ : At sufficiently high- $n$ the resonant contribution (DR) is

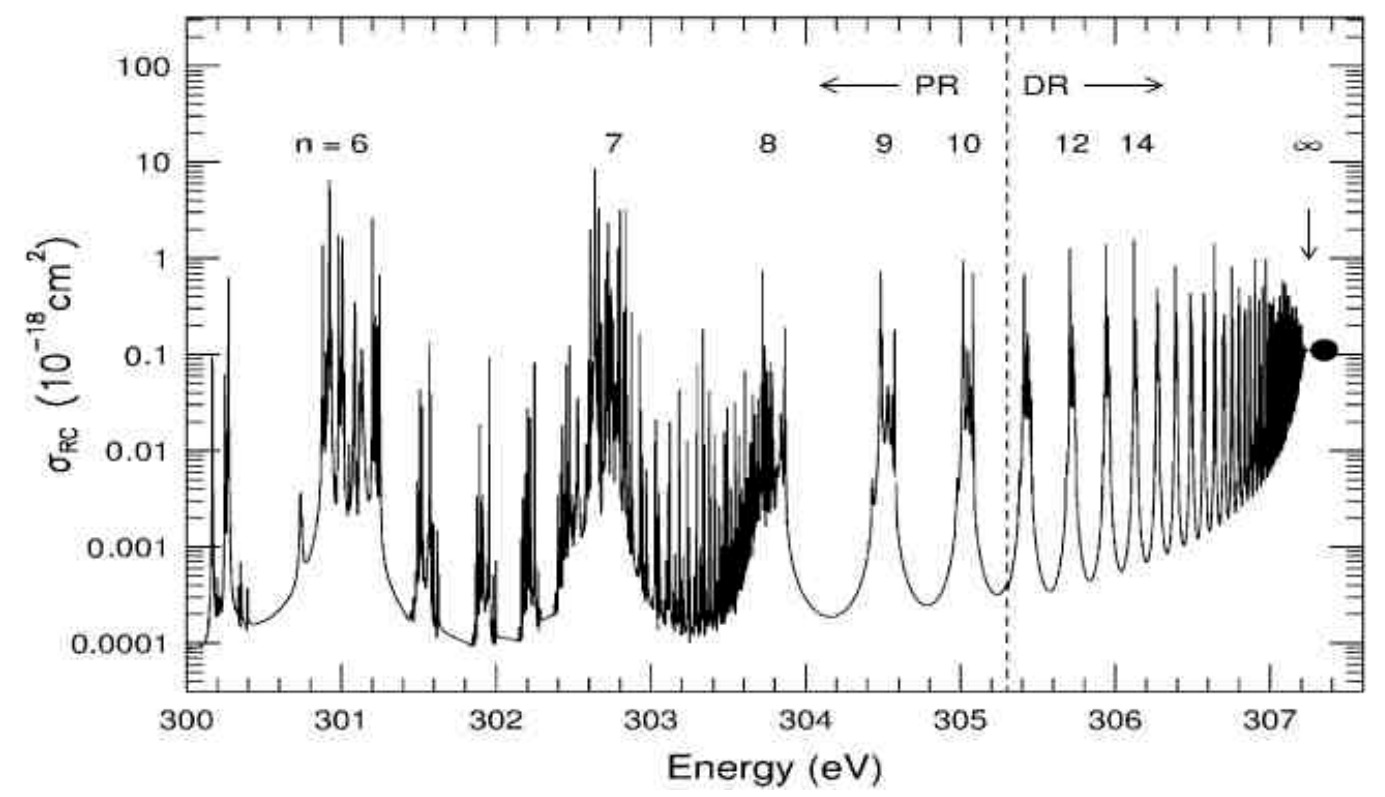

Fig. 9. Photo-recombination (PR), DR, and excitation cross sections (Zhang et al., 1999), as derived from photoionization calculations (left of the dashed line), and the dielectronic (DR) cross sections (right of the dashed line) for $(e+C V) \rightarrow C$ IV; the filled circle represents the near-threshold value of electron impact excitation cross section for the dipole transition $1{ }^{1} S_{0}-2{ }^{1} P_{1}^{0}$ in $\mathrm{C} \mathrm{V}$.

very large compared to the background, non-resonant photorecombination (PR) cross section. In the unified method of electron-ion recombination, for $n>n_{\max }$; we employ the BS theory to compute the detailed and the averaged resonant DR cross sections; the background contributions are computed in the hydrogenic approximation. The agreement and the continuity between the three sets of data in Fig. 9 shows the CC method for electron-ion recombination to be accurate and self-consistent with inverse photo-ionization (photorecombination) and electron impact excitation.

Another example that demonstrates the threshold behavior and continuity of DR and electron impact excitation cross sections is the recent calculation for (e + C IV) $\rightarrow$ C III (Pradhan et al., 2001a). In Fig. 10 we delineate the fine structure $\sigma_{D R}$ in the energy region spanned by the fine structure ${ }^{2} P^{0}{ }_{-1 / 2,3 / 2}$ thresholds. Fig. 10(a) shows the detailed resonances in the vicinity of the two series limits. Fig. 10(b) shows the $\sigma_{D R}$ averaged over the lower resonance series ${ }^{2} P^{0}{ }_{12}^{0} n \ell$ below the ${ }^{2} P_{12}^{0}$ level, but still with the detailed resonance structures due to the higher series ${ }^{2} P^{0}{ }_{32} n \ell$ (solid line). The $\sigma_{\mathrm{DR}}$ averaged over both series is shown as the dashed line. Above the ${ }^{2} P^{0}{ }_{1 / 2} ; \sigma_{\mathrm{DR}}$ is averaged over the ${ }^{2} P^{0}{ }_{32} n \ell$ series. The sharp drop in the total $\sigma_{\mathrm{DR}}$ at the ${ }^{2} P^{0}{ }_{1 / 2}$. threshold reflects the termination of DR due to the ${ }^{2} P^{0}{ }_{1 / 2}^{0} n \ell$ resonance series, and with the ${ }^{2} P^{0}{ }_{32} n \ell$ contribution still low in spite of the fact that $n \approx 96$. The large drop in the DR cross section is due to enhanced autoionization into the excited level, when the ${ }^{2} P^{0}{ }_{1 / 2}^{0} n \ell$ channel opens up at the lower fine structure threshold ${ }^{2} P_{\cdot 1 / 2}^{0}$ while the radiative decay 

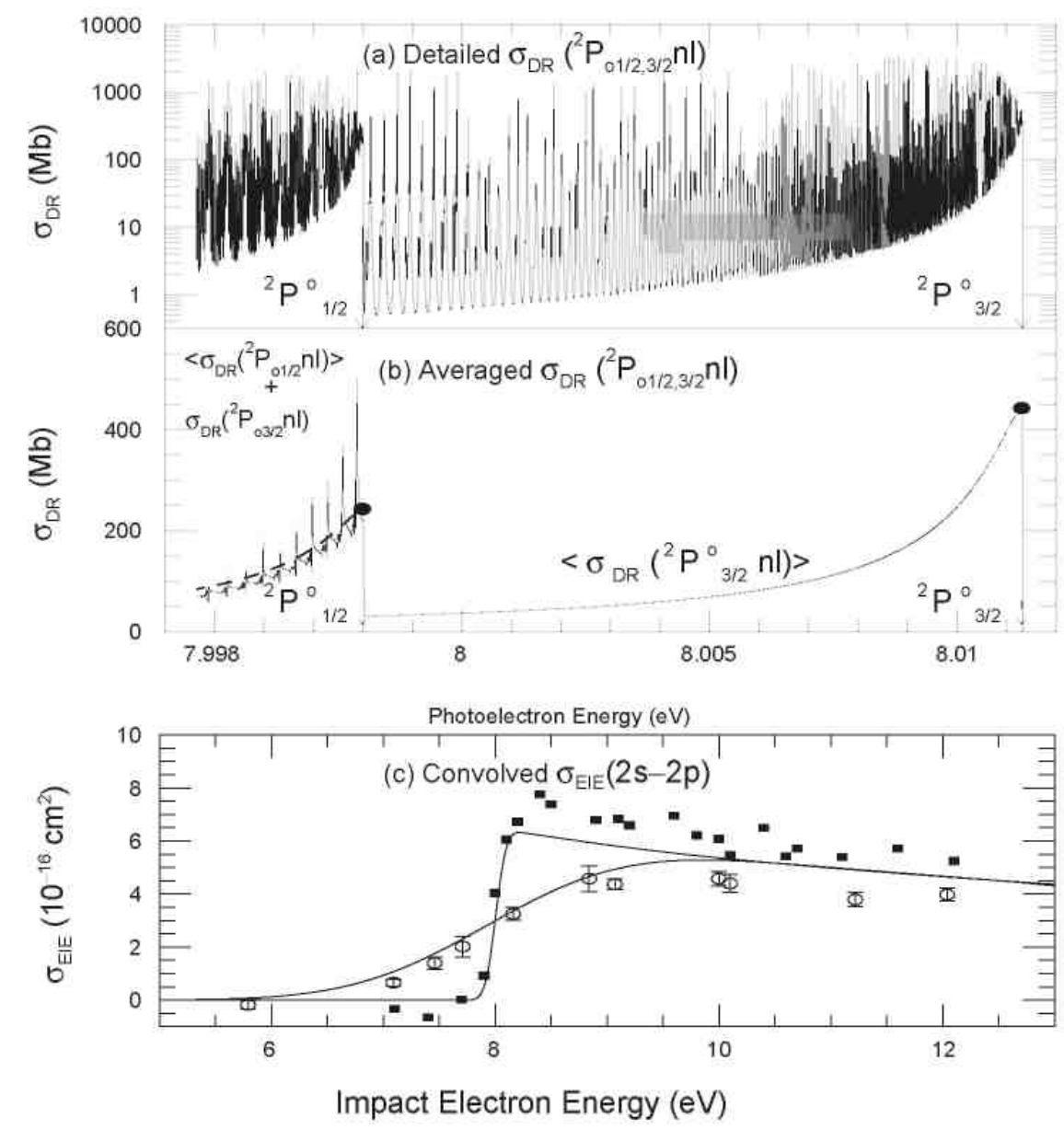

Fig. 10. DR and electron impact excitation cross sections $\sigma_{\mathrm{DR}}$ and $\sigma(\mathrm{EIE})$ of C IV: (a) detailed $\sigma_{\mathrm{DR}}$. with ${ }^{2} P^{0}{ }^{0}{ }_{2.3 / 2}$ resonances; (b) $\sigma_{\mathrm{DR}}$ averaged over ${ }^{2} P_{1_{1 / 2}}^{o} \mathrm{n} \ell$ and detailed ${ }^{2} P_{32}^{0} n \ell$ resonances (solid line), average over the ${ }^{2} P_{3}^{0}{ }_{32}^{o} n \ell$ (dashed line); the dark circles are the peak averaged $\sigma_{\mathrm{DR}}$; (c) $\sigma$ (EIE) convolved over experimental data with FWHM = 0:175 eV from Greenwood et al. (1999, filled squares), and with FWHM = 2:3 eV from Janzen et al. (1999, open circles).

remains constant. The $\sigma_{\mathrm{DR}}\left(P_{3 / 2}^{0} n \ell\right)$ contribution builds up to the second peak at ${ }^{2} P^{0}{ }_{322}$. In Fig. 10(b) it is shown that the resonance averaged $\lim _{n} \rightarrow \infty\left\langle\sigma_{\mathrm{DR}}\left({ }^{2} P_{1 / 2}^{0} n \ell\right\rangle=242: 57 \mathrm{Mb}\right.$ (dark circle at $\left.{ }^{2} P_{1 / 2}^{0}\right)$, but the detailed $\sigma_{\mathrm{DR}}$ has resonances due to the higher series $\left({ }^{2} P^{0}{ }_{32}^{0} n \ell\right)$ lying at and near threshold. The resonance averaged $\sigma_{\mathrm{DR}}$ at the next DR peak, $\lim _{n \rightarrow \infty} \rightarrow\left\langle\sigma_{\mathrm{DR}}\left({ }^{2} P_{32}^{0} n \ell\right)\right\rangle$ $=441: 81 \mathrm{Mb}$ (dark circle at $\left.{ }^{2} P^{0}{ }_{32}\right)$. Interestingly, the fine structure in the theoretical $\sigma_{\mathrm{DR}}$ in Fig. 10(a) and (b) appears to be discernible as a small dip in experimental data in just below $8 \mathrm{eV}$ (Schippers et al., 2001; Pradhan et al., 2001a). Although the ${ }^{2} P^{0}{ }_{1 / 2,3 / 2}$ separation is only 0:013 eV; it may be possible to detect these fine structure threshold effects in future experiments with increased resolution.

At the ${ }^{2} P_{1 / 2,3 / 2}^{0}$ thresholds the sum of the averaged fine structure $\left\langle\sigma_{\mathrm{DR}}\right\rangle=\sigma_{\mathrm{EIE}}=684: 38 \mathrm{Mb}$ : Fig. 10(c) compares the near-threshold EIE cross sections with the absolute measurements from two recent experiments, (Greenwood et al., 1999; Janzen et al., 1999), convolved over their respective beam widths of 0.175 and 2:3 eV: Our results are in good agreement with both sets (and 
also with another recent experiment by Bannister et al. (1998). Although the present results are the first BPRM calculations with relativistic fine structure for C IV, their sum is in good agreement with previous LS coupling CC calculations of $\sigma_{\text {EIE }}$ reported by Burke (1992), Griffin et al. (2000) and Janzen et al. (1999).

\subsection{Multiple DR bumps in $\alpha_{R}(T)$}

The general shape of the unified ( $\mathrm{e}+$ ion) recombination rate coefficient $\alpha_{R}(T)$ is illustrated in examples in the previous section. Basically, one finds an exponential decrease with temperature starting with the background RR part of the rate coefficient, attenuated by a large bump at higher temperatures due to DR. However, resonance series due to several ion thresholds might contribute to $\alpha_{R}(T)$ over extended energy ranges. Nussbaumer and Storey (1983) first pointed out that near-threshold low-energy resonances give rise to a low-temperature DR bump. An extension of the same concept is seen if several groups of resonances are interspersed throughout, from low to high energies. In such cases, we find multiple DR bumps in $\alpha_{R}(T)$ : Fig. 11 shows (e + Fe XXII) $\rightarrow$ Fe XXI recombination, with discernible bumps due to the $n=2$ and 3 groups of resonances (Nahar, 2004a).

The R-matrix eigenfunction CC expansion for B-like Fe XXII includes 29 LS terms (29-CC) up to the $n=3$ complex of configurations: $2 s^{2} 2 p ; 2 s 2 p^{2} ; 2 p^{3} ; 2 s 2 p 3 s$;

$2 s 2 p 3 p ; 2 s 2 p 3 d ; 2 s^{2} 3 s ; 2 s^{3} 2 p ; 2 s^{2} 3 d$ : Including only the $n=2$ terms gives an 8-CC expansion. Fig. 12 shows the detailed photoionization cross sections for two excited states, out of a total of 835 states of Fe XXI calculated using the 29-CC Fe XXII target expansion

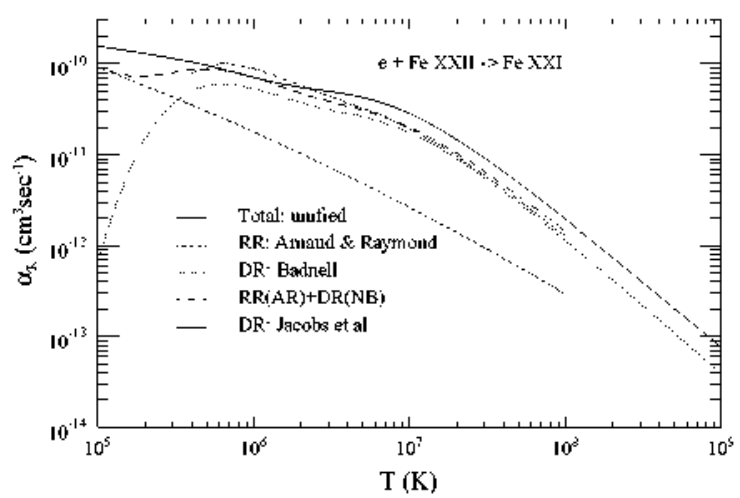

Fig. 11. Multiple DR bumps in the (e + Fe XXII) $\rightarrow$ Fe XXI recombination rate coefficients (Nahar, 2004a), compared with previous RR and DR values (Arnaud and Raymond, 1992; Badnell, 1986; Jacobs et al., 1980). The multiple bumps in $\alpha_{R}(T)$ are due to DR via several groups of resonances in different energy regions in Fig. 12.

(the highest 8-CC threshold is also marked). It is particularly noteworthy how the same resonance complexes appear quite differently in photoionization of states with different angular and spin symmetries $S L \pi$ : It is clear that $\Delta n>0$ transitions in the core ion play an important role in the determination of total $\alpha_{R}(T)$ in Fig. 11 throughout the temperature range where Fe XXI is likely to be abundant in laboratory and astrophysical plasmas, and agree fairly with the recently reported experimental values in the "photoionized zone" $T \sim 30-80$ eV (Savin et al., 2004b). At very low energies there are additional resonance structures which may not be of practical importance but provide a good check on theoretical works; more detailed calculations in this region $(<20 \mathrm{eV})$ are in progress. However, at temperatures $T>10^{6} \mathrm{~K}(\sim 100 \mathrm{eV})$ the present rate coefficients in Fig. 11 
are considerably higher than those reported by Savin et al. (2004b), since their measurements do not extend to high energies relevant to collisionally ionized plasmas, particularly the multiple DR bump region described herein; the temperature of maximum abundance of Fe XXI in collisional equilibrium is $\sim 10^{7} \mathrm{~K}$ (Arnaud and Raymond, 1992). A more detailed comparison will be reported elsewhere, together with total $(\mathrm{e}+\mathrm{ion})$ recombination rate coefficients for Fe XXI at all temperatures of general importance (see also Section 4.6 on ionization equilibrium).

\subsection{Radiation damping of low-n resonances}

Scattering, photoionization, and recombination of all atomic systems involve an infinite number of resonances. Close coupling calculations allow for resonances in an ab initio manner. Broad low-lying near-threshold resonances have autoionization rates $\Gamma^{\mathrm{a}} \sim 10^{14} \mathrm{~s}^{-1}$;

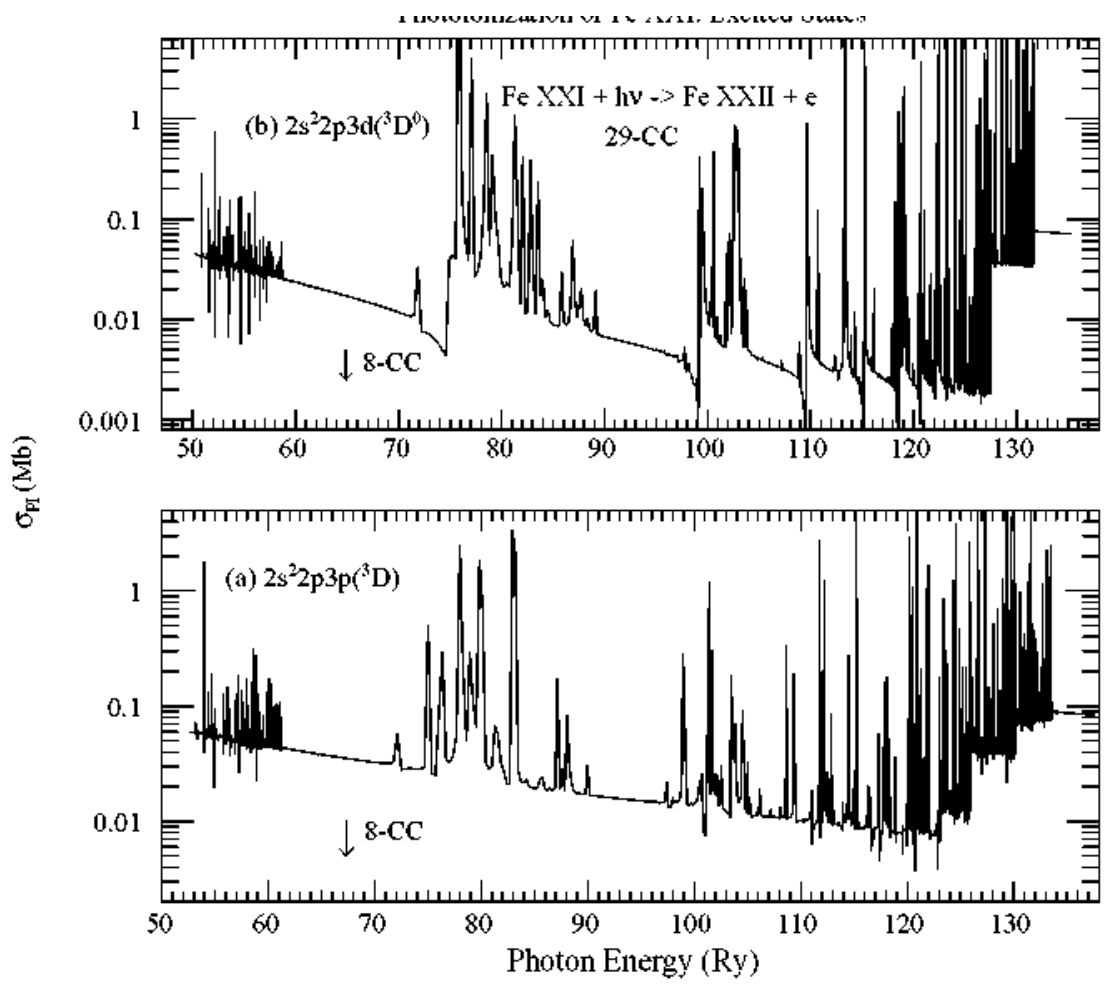

Fig. 12. Photoionization cross sections for two excited states of carbon-like Fe XXI, leading to multiple bumps in $O . R(T)$ in Fig. 11 (Nahar, 2004b). The 8-CC refers to the $n=2$ thresholds, whereas the 29-CC refers to the target states of Fe XXII up to $n=3$ in the R-matrix CC expansion.

much greater than typical dipole radiative decay rates $\Gamma^{\mathrm{r}} \sim 10^{6-12} \mathrm{~s}^{-1}$. For most atomic systems up to the iron-peak elements ( $\mathrm{Z}=30), \Gamma^{\mathrm{r}}<10^{12} \mathrm{~s}^{-1}$ for the lowest dipole transitions. Therefore, a priori, radiation damping is not expected to be a dominant effect. Notable exceptions are high- $Z$, H-like and He-like ions with $\Gamma^{\mathrm{r}} \sim \Gamma^{\mathrm{a}}$. For highly excited states with $n \rightarrow \infty$, the $\Gamma^{\mathrm{a}}$ decreases as $n^{-3}$. and $\Gamma^{\mathrm{r}}$. dominates.

However, all resonances are susceptible to radiation damping somewhere between $0-100 \%$, i.e. damping ratios (undamped/damped) from 1 to $\infty$, depending on $n, \ell$, energy relative to threshold, and radiative transition probability $\Gamma^{\mathrm{r}}$. (radiation damping of resonance profiles was 
demonstrated in Pradhan, 1981, and Pradhan and Seaton, 1985). However, determination of damping factors of individual resonances per se may be inaccurate as they depend on the precise positions, heights, and shapes of resonances. Undamped/damped ratios > unity for a few individual resonance profiles do not imply that radiation damping is important in general. More meaningful than the damping factors for individual resonances is the combined effect of all damped resonances on the integrated rate coefficient for an atomic process, i.e. on the quantities of practical interest in laboratory and astrophysical applications. Rate coefficients are usually obtained by averaging cross sections over a Maxwellian. Generally broad near-threshold resonances corresponding to the lowest $n$ and $\ell$ make the dominant contribution, relative to narrower ones with higher $n$ and $\ell$. The CC calculations include all closed channels with $n \leq n_{o}$ and $\ell \leq n-1$. Other methods based on quantum defect theory, such as Gailitis averaging of resonances, or the BS theory of DR, are employed for $n_{o}<n \leq \infty$. As mentioned earlier, only the H-like or the He-like core ions appear to require a detailed consideration of damping of low- $n\left(n<n_{o}\right)$ resonances.

The primary application of the present CC method for $(e+$ ion) recombination is to obtain unified total recombination rates for elements up to the iron-peak elements with $Z \leq 30$, well within the validity of the intermediate coupling approximation using the BP method. In general, the contribution from the near-threshold region is dominated by large resonances with $\Gamma^{\mathrm{a}} \sim 10^{14} \mathrm{~s}^{-1}$; the extremely narrow resonances, with $\Gamma^{\mathrm{a}}$ several orders of magnitude smaller, make relatively little contribution and may be neglected. The calculations generally resolve each resonance $n$-complex up to $\ell \leq 4$; the higher $\ell$ resonances are assumed to be damped out, thereby making allowance for radiation damping, although it is not a large effect on the final results. It is interesting to note that experimental measurements also retain up to about $\ell \leq 4$ levels before field ionization (Wolf et al., 1991). With the exception of $\mathrm{H}$ - and He-like ions where all resonances undergo significant radiative damping, the cross sections and rate coefficients should not be influenced by radiation damping when the core radiative transitions do not significantly compete with autoionization. This is certainly true for all $\Delta n=0$ radiative core transitions even in highly charged ions. For example, the theoretical BPRM results without radiation damping of low- $n$ resonances in photorecombination cross sections for (e + Ar XIV) recombintion with a Boron-like core transition 2s-2p (Zhang and Pradhan, 1997), are in excellent agreement with the absolute cross sections measured from the heavy ion storage ring CRYRING in Stockholm (DeWitt et al., 1996), both in magnitude and details of the extensive resonance structures and background cross section.

\subsection{Resolution and analysis of resonance structures}

The present unified approach incorporates the major effects of importance in the calculation of total effective recombination cross sections and rate coefficients. However, without loss of generality, some other physical effects might be of marginal interest in exceptional cases. Criticism of the shortcomings of the method are confined to isolated resonances, series limits, or small energy ranges, but which otherwise affect the rate coefficients by no more than a few percent (Gorczyca et al., 2002). For example, the precise choice of $n_{0}$. where the background recombination cross section becomes negligible and DR dominates for $n>n_{0}$; is not crucial and may be varied. Although some low- $n$ resonances close to $n_{0}$ might be very narrow and not fully resolved, the effect is barely discernible (Zhang et al., 2001).

The precise positions, shapes, and heights of resonances depend not simply on resolution, but also on the CC wavefunction expansions (and accuracy thereof), relativistic fine structure, 
parameters chosen for $R$-matrix calculations, and numerical inaccuracies. Resolution per se is not a major problem by comparison (cf. Ramirez and Bautista, 2002). The relevant radiative decay rates in neutrals, near-neutrals, and many-electron systems are far smaller than the autoionization rates of near-threshold resonances. For example, our detailed analysis for Fe XVII (Pradhan et al., 2001b) shows that radiation damping is altogether unimportant.

Finally, it might be pointed out that, as yet, no total recombination rate coefficients of high accuracy have been presented in literature that are discrepant by about more than $10 \%$ (the best estimate of uncertainty in theory or experiments), due to radiation damping or other effects, with those calculated using the unified formulation. The narrow resonance structures may differ owing to resolution and accuracy of their energies, widths, heights, and shapes. The low- $n, \ell$ resonances $\left(n<n_{o} \approx 10\right)$ are usually highly resolved in the reported unified calculations and adequately treated by the perturbative method employed. Extremely narrow low- $n, \ell$ resonances that might remain unresolved should be damped out, or have negligible effect.

\subsubsection{Plasma effects}

While the unified method is quite general in scope, it does not incorporate external field and density effects. These might be important in practical situations, such as for DR near series limits. For example, in Fig. 3 the field-ionization cut-off in the experiment is estimated at $n_{\mathrm{F}} \approx 19$ (Schippers et al., 2001). The theoretical results used the same approximate value for comparison (Pradhan et al., 2001a). However, a suitably general treatment of plasma fields and densities for the calculation of total $(\mathrm{e}+\mathrm{ion})$ recombination cross sections in the present formulation is yet to be implemented.

\subsection{Ionization equilibrium}

The present approach is especially suited to the calculation of ionization fractions in astrophysical sources such as H II regions in general: diffuse and planetary nebulae, supernova remnants, and broad line regions of active galactic nuclei. Ionization balance is the prime feature of astrophysical models of these objects. The two most common assumptions for the ionization conditions in the plasma are: (i) photoionization equilibrium, and (ii) collisional or coronal equilibrium (Osterbrock, 1989). The dominant ionizing process in the first case is photoionization from the radiation field in the source, and in the second case, electron impact ionization in usually optically thin plasmas (such as the solar corona). Both ionization processes are sought to be balanced by the inverse process of electron-ion recombination in the ambient medium characterized by a given electron temperature, usually in terms of a Maxwellian distribution, and an electron density. The two sets of ionization balance equations may be written as

$$
\begin{aligned}
& \int_{v_{0}}^{w} \frac{4 \pi J_{v}}{h v} N\left(X^{2}\right) \sigma_{\mathrm{PI}}\left(v, X^{2}\right) \mathrm{d} v \\
& \quad=\sum_{j} N_{e} N\left(X^{z+1}\right) \varkappa_{\mathrm{R}}\left(X_{j}^{z} ; T\right) \\
& \text { and } \\
& C_{\mathrm{I}}\left(T, X^{z}\right) N_{\mathrm{e}} N\left(X^{2}\right)=\sum_{i} N_{e} N\left(X^{2+1}\right) \varkappa_{\mathrm{R}}\left(X_{j}^{2} ; T\right),
\end{aligned}
$$

where $\alpha_{\mathrm{R}}\left(X_{j}^{Z}, T\right)$ is the total electron-ion recombination rate coefficient of the recombined ion of charge $z, X_{j}^{z}$, to state $j$ at electron temperature $T, C_{\text {II }}$ is the rate coefficient for electron impact 
ionization, and $\sigma_{\mathrm{PI}}$ is the photoionization cross section evaluated at photon frequency $v$ and convoluted with the isotropic radiation density $J_{\mathrm{v}}$ of the source; $N_{e}, N\left(X^{\mathrm{Z}+1}\right)$, and $N\left(X^{\mathrm{Z}}\right)$ are the densities for the free electrons, and the recombining and recombined ions, respectively.

Implicit in these equations is the assumption that the ionization rates on the left hand side refer to the ground state of the ion. This condition is predicated on the assumption that the radiative and collisional processes proceed on faster time scales than photoionization and recombination. Substantial departures from these equilibrium conditions may result at high densities where some excited states are significantly populated or in LTE. The sum on the right hand side extends over the infinite number of excited states into which recombinations can take place, depending on the temperature.

Unlike previous works, the present work seeks to satisfy the photoionization equilibrium condition (Eq. (1)) in a fundamentally consistent manner: the photoionization and recombination calculations are carried out using the same set of atomic eigenfunctions. Furthermore, as the detailed photoionization cross sections include autoionizing resonances in an ab initio manner, the electron-ion recombination rates subsume both the radiative and the dielectronic recombination processes, that have been treated separately in previous works using different methods.

In the coronal approximation (Eq. (2)) some of the previous calculations for ionization fractions are: Jacobs et al. (1980, and reference therein), Shull and van Steenberg (1982), Arnaud and Raymond (1992), Sutherland and Dopita (1993). In general, most of the electron-ion recombination data employed in the earlier works is based on radiative recombination (RR) rates (e.g. Aldrovandi and Pequignot, 1973) derived from photoionization cross sections that neglect the now well-established phenomenon of autoionizing resonances (e.g. Reilman and Manson, 1979), and on dielectronic

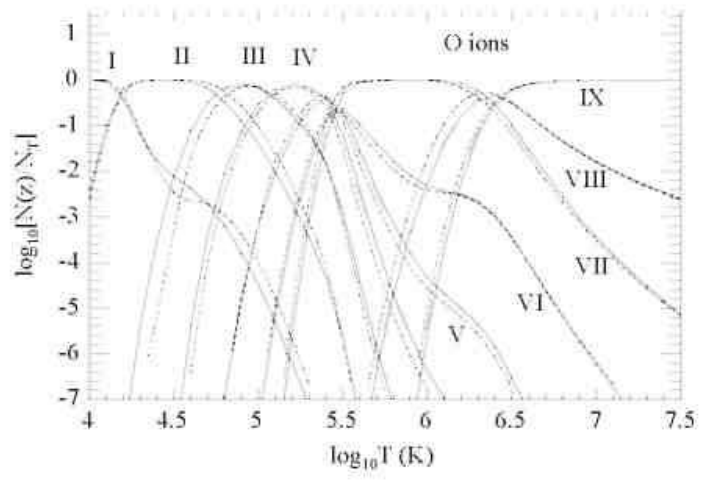

Fig. 13. Ionization fractions of oxygen ions in coronal or collisional equilibrium (solid line, Nahar, 1999) using unified (e + ion) recombination rate coefficients, compared to previous values (dashed line, Sutherland and Dopita, 1993).

recombination (DR) rates derived from the Burgess general formula (Burgess, 1965), but incorporating the important advances made in the treatment of DR by Jacobs et al (1977), who showed the effect of auto-ionization into excited states, and later by Nussbaumer and Storey (1983) who established the significant contribution from near-threshold autoionizing resonances resulting in a low-temperature bump (in addition to the usual high-temperature rise in the DR rate).

Using the present unified rate coefficients, ionization fractions for a number of elements have been computed (e.g. Nahar and Pradhan, 1997 for C and N, Nahar, 1999 for O). Fig. 13 shows a sample calculation in coronal equilibrium (Nahar, 1999). Ongoing calculations for astrophysically abundant elements are being reported in a continuing series of publications in the 
Astrophysical Journal Supplements (e.g. Nahar and Pradhan, 1997).

\section{Summary}

The electron-ion recombination process is unified in nature; the non-resonant and resonant contributions are inseparable and observed or measured together. However, these are usually treated independently as radiative and dielectronic recombination (RR and DR). A Unified Approach, based on the close-coupling approximation and the R-matrix method, has been developed and applied to the calculation of total recombination cross sections and rates for over 50 atoms and ions.

The ab initio calculations employ a coupled eigen-function expansion for detailed photoionization cross sections, with extensive delineation of resonance structures, for a large number of bound levels of the (e + ion) system, up to $n(S L J)=n_{o} \approx 10$. Detailed balance (Milne relation) thereupon yields photorecombination cross sections at all energies. For $n_{0}<n \leq \infty$, recombination is predominantly DR and is treated using the precise theory of Bell and Seaton; the non-resonant background contribution is treated hydrogenically as "top-up" contribution.

The advantages of the R-matrix method for $(\mathrm{e}+\mathrm{ion})$ recombination are

1. Unified treatment of non-resonant and resonant processes (RR and DR) in an ab initio manner.

2. Self-consistent treatment of photoionization and recombination with identical wavefunction expansions.

3. Relativistic fine structure effects are included using the Breit-Pauli R-matrix method.

4. High-resolution of resonances to arbitrary accuracy, including radiation damping when necessary (e.g. Hand He-like ions).

5. Detailed agreement with experimental cross sections measured at synchrotron ion storage rings to $10-20 \%$.

6. General validity for all ionization states of elements, from neutrals to highly ionized.

7. Total (e+ion) recombination rate coefficients are obtained at all temperatures for practical applications.

8. Level-specific rate coefficients are obtained for a large number of levels (usually hundreds) up to $n(S L J)-n_{o} \approx 10$.

9. Ionization fractions of elements in plasmas may be computed using consistent and accurate photoionization cross sections and total recombination rate coefficients.

10. Recombination spectra may be computed using level-specific recombination rate coefficients; recombination-cascade matrices may be derived using transition probabilities that may also be computed with the same R-matrix CC wavefunction expansion as photoionization, recombination, and excitation.

The unified method overcomes the shortcomings of simpler methods based on independent resonance approximation that (i) divide (e + ion) recombination into RR and DR, usually computed in different approximations, (ii) further subdivision of DR into low- and high-energy parts, such as $\Delta n=0$, and $\Delta n=1$, etc., and (iii) neglect interference between non-resonant and resonant components, likely to be of importance for neutrals and other ionization stages with strong coupling effects, thereby limiting their validity to highly charged ions.

The primary aim of this work is to obtain precise and complete total recombination rates for practical applications. Following is the list of atoms and ions for which self-consistent sets of $\sigma_{\mathrm{PI}}$ and $\alpha_{\mathrm{R}}(T)$ have so far been obtained for over 50 ions (e.g. Nahar and Pradhan, 1997; 
http://www.astronomy.ohio-state.edu/ pradhan):

Carbon: C I, C II, C III, C IV, C V, C VI.

Nitrogen: N I, N II, N II, N IV. N V. N VI, N VI.

Oxygen: O I. O II. O III. O IV, O V, O VI. O VII. O

VII.

Si: Si I, Si II, Si IX.

$S$ : S II, S III, S XI.

Ar: Ar V, Ar XIII.

$\mathrm{Ca}$ : Ca VII, Ca XV.

$F e:$ Fe I. Fe II. Fe III. Fe IV, Fe V, Fe XIII, Fe XVII.

Fe XXI, Fe XXIV, Fe XXV, Fe XXVI.

Ni: Ni II, Ni XXVI.

C-like: F IV, Ne V, Na VI, Mg VII, Al VIII.

Li-like: in progress.

The datasets for each ion include level-specific unified recombination rate coefficients for typically hundreds of bound levels with $n \leq 10$ : The self-consistent sets of photoionization /recombination datasets also include new photoionization cross sections that are generally an improvement over the earlier Opacity project data (The Opacity Project, 1995/1996), since more extensive and accurate eigenfunction expansions are employed. R-matrix transition probabilities are also available for many of the ions listed.

\section{References}

Aldrovandi, S.M.V., Pequignot, D., 1973. Astron. Astrophys. 25, 137.

Arnaud, M., Raymond, J., 1992. Astrophys. J. 398, 394.

Badnell, N.R., 1986. J. Phys. B 19, 3827.

Badnell, N.R., Pindzola, M.S., Griffin, D.C., 1990. Phys. Rev. A 41, 2422.

Bannister, M.E., Chung, R.-S., Djuric, N., Wallbank, B., Woiteke, O., Zhou, S., Dunn, G.H., Smith, A.C.H., 1998. Phys. Rev. A 57, 278.

Bates, D.R., Massey, H.S.W., 1943. Philos. Trans. R. Soc. A 239, 269.

Bautista, M.A., 1996. Astron. Astrophys. Suppl. 119, 105.

Bautista, M.A., Pradhan, A.K., 1995. J. Phys. B 28, L173.

Bautista, M.A., Pradhan, A.K., 1997. Astron. Astrophys. Suppl. 126, 365.

Beiersdorfer, P., Philips, T.W., Wong, K.L., Marrs, R.E., Vogel, D.A., 1992. Phys. Rev. A 46, 3812.

Bell, R.H., Seaton, M.J., 1985. J. Phys. B 18, 1589.

Berrington, K.A., Burke, P.G., Butler, K., Seaton, M.J., Storey, P.J., Taylor, K.T., Yu, Yan, 1987. J. Phys. B 20, 6379.

Berrington, K.A., Eissner, W.B., Norrington, P.H., 1995. Comput. Phys. Commun. 92, 290.

Burgess, A., 1965. Astrophys. J. 141, 1588.

Burgess, A., Seaton, M.J., 1960. Mon. Not. R. Astron. Soc. 120, 121.

Burke, V.M., 1992. J. Phys. B 25, 4917.

Burke, P.G., Berrington, K.A., 1993. Atomic and Molecular Processes: An R-matrix Approach. Institute of Physics Publ., Bristol.

Burke, P.G., Robb, W.D., 1975. Adv. At. Mol. Phys. 11, 143.

Champeaux, J.-P., Bizau, J.-M., Cubaynes, D., Blancard, C., 
Nahar, S.N., Hitz, D., Bruncau, J., Wuilleumier, F.J., 2004a. Astrophys. J. Suppl. 148, 583.

Covingtion, et al., 2001. Phys. Rev. Lett. 87, 243002.

Davies, P.C.W., Seaton, M.J., 1969. J. Phys. B 2, 757.

DeWitt, D.R., Schuch, R., Zong, W., Asp, S., Biederman, C.,

Chen, M.H., Badnell, N.R., 1996. Phys. Rev. A 53, 2327.

Eissner, W., Jone, S.W., Nussbaumer, N., 1974. Comput. Phys.

Commun. 8, 270.

Gharaibeh, et al., 2003, Bull. Am. Phys. Soc. 43(3) JI 90, 94.

Gorczyca, T.W., Badnell, N.R., Savin, D.W., 2002. Phys. Rev. A 65, 062707.

Greenwood, J.B., Smith, S.J., Chutjian, A., 1999. Phys. Rev. A 59, 1348.

Griffin, D.C., Badnell, N.R., Pindzola, M.S., 2000. J. Phys. B 33, 1013.

Hahn, Y., 1985. Adv. At. Mol. Phys. 21, 123.

Hahn, Y., Lagattuta, K.J., 1988. Phys. Rep. 166, 195. Hummer, D.G., 1994. Mon. Not. R. Astron. Soc. 268, 109.

Hummer, D.G., Berrington, K.A., Eissner, W., Pradhan, A.K., Saraph, H.E., Tully, J.A., 1993. Astron. Astrophys. 279, 298.

Jacobs, V.L., Davis, J., Keppler, P.C., Blaha, M., 1977. Astrophys. J. 215, 690.

Jacobs, V.L., Davis, J., Rogerson, J.E., Blaha, M., Cain, J., Davis, J., 1980. Astrophys. J. 239, 1119.

Janzen, P.H., Gardner, L.D., Reisenfield, D.B., Savin, D.W., Kohl, J.L., 1999. Phys. Rev. A 59, 4821.

Kelly, H.P., 1972. Phys. Rev. A 6, 1048.

Kilgus, G., et al., 1990. Phys. Rev. Lett. 64, 737.

Kilgus, G., Habs, D., Schwalm, D., Wolf, A., Schuch, R., Badnell, N.R., 1993. Phys. Rev. A 47, 4859.

Kjeldsen, H., Folkmann, F., Hensen, J.E., Knudsen, H., Rasmussen, M.S., West, J.B., Andersen, T., 1999. Astro-phys. J. 524, L143.

Kjeldsen, H., Kristensen, B., Folkmann, F., Andersen, T., 2002. J. Phys. B 35, 3655.

Mannervik, S., DeWitt, D.R., Engstrom, L., Lidberg, J., Schuch, R., Zhong, W., 1998. Phys. Rev. Lett. 81, 313.

Nahar, S.N., 1996a. Phys. Rev. A 53, 1545.

Nahar, S.N., 1996b. Phys. Rev. A 53, 2417.

Nahar, S.N., 1997. Phys. Rev. A 55, 1980.

Nahar, S.N., 1999. Astrophys. J. Suppl. 120, 131.

Nahar, S.N., 2000. Astrophys. J. Suppl. 126, 537.

Nahar, S.N., 2002. Phys. Rev. A 65, 052705.

Nahar, S.N., 2004a. Phys. Rev. A, submitted.

Nahar, S.N., 2004b. in preparation.

Nahar, S.N., Bautista, M.A., 1999. Astrophys. J. 120 (Suppl.), 327.

Nahar, S.N., Pradhan, A.K., 1992. Astrophys. J. 397, 729. Nahar, S.N., Pradhan, A.K., 1994a.

Phys. Rev. A 49, 1816.

Nahar, S.N., Pradhan, A.K., 1994b. J. Phys. B 27, 429.

Nahar, S.N., Pradhan, A.K., 1995. Astrophys. J. 447, 966.

Nahar, S.N., Pradhan, A.K., 1997. Astophys. J. Suppl. 111, 339.

Nahar, S.N., Bautista, M.A., Pradhan, A.K., 1997. Astrophys. J. 479, 497. 
Nahar, S.N., Bautista, M.A., Pradhan, A.K., 1998. Phys. Rev. A 58, 4593.

Nussbaumer, H., Storey, P.J., 1983. Astron. Astrophys. 126, 75.

Osterbrock, D.E. 1989. in: Astrophysics of Gaseous Nebulae and Active Galactic Nuclei. University Science Books, California.

Pindzola, M.S., Badnell, N.R., Griffin, D., 1990. Phys. Rev. A 42, 282.

Pradhan, A.K., 1981. Phys. Rev. Lett. 47, 79.

Pradhan, A.K., 1983a. Phys. Rev. A 28, 2113.

Pradhan, A.K., 1983b. Phys. Rev. A 28, 2128.

Pradhan, A.K., Seaton, M.J., 1985. J. Phys. B 18, 1631.

Pradhan, A.K., Zhang, H.L., 1997. J. Phys. B 30, L571.

Pradhan, A.K., Chen, Guo Xin, Nahar, S.N., Zhang, H.L., 2001a. Phys. Rev. Lett. 87, 183201.

Pradhan, A.K., Nahar, S.N., Zhang, H.L., 2001b. Astrophys. J. Lett. 549, L265.

Presnyakov, L.P., Urnov, A.M., 1975. J. Phys. B 8, 1280.

Ramirez, J.M., Bautista, M.A., 2002. J. Phys. B 35, 4139.

Reilman, R.F., Manson, S.T., 1979. Astrophys. J. Suppl. 40, 815.

Sakimoto, K., Terao, M., Berrington, K.A., 1990. Phys. Rev. A 42, 291.

Sampson, D.H., Zhang, H.L., 1995. J. Quant. Spectrosc. Radiat. Transfer 54, 345.

Sampson, D.H., Zhang, H.L., Mohanty, A.K., Clark, R.E.H., 1989. Phys. Rev. A 40, 604.

Savin, et al., 1999. Astrophys. J. Suppl. 123, 687.

Savin, D.W., Kahn, S., Gwinner, G., Grieser, M., Repnow, R., Saathoff, G., Schwalm, D., Wolf, A., Müller, A., Schippers, S., Zavodszky, P., Chen, M.H., Gorczyca, T.W., Zatsar-inny, O., Gu, M.F., 2004b. Astrophys. J. Suppl. 147, 421.

Seaton, M.J., 1987. J. Phys. B 20, 6363.

Seaton, M.J., Storey, P.J., 1976. In: P.G. Burke, B.L. Moiseiwitsch, (Eds.), Atomic Processes and Applications, North-Holland, Amsterdam; p. 133.

Schippers, S., Bartsch, T., Brandau, C., Muller, A., Linkemann, J., Saghiri, A.A., Wolf, A., 1999. Phys. Rev. A 59, 3092.

Schippers, S., Müller, A., Gwinner, G., Linkemann, J., Saghiri, A.A., Wolf, A., 2001. Astrophys. J. 555, 1027.

Shull, J.M., van Steenberg, M., 1982. Astrophys. J. Suppl. 48, 95.

Storey, P.J., Hummer, D.G., 1992. Comput. Phys. Commun. 66, 129.

Sutherland, R.S., Dopita, M.A., 1993. Astrophys. J. Suppl. 88, 253.

The Opacity Project Team. (1995/1996). The Opacity Project, Vol. 1, 1995; Vol. 2, 1996, Institute of Physics Publishing, Bristol, UK.

Trefftz, E., 1983. J. Phys. B 16, 1247.

Verner, D.A., Yakovlev, D.G., Band, I.M., Trzhaskovskaya, M.B., 1993. At. Data Nucl. Data Tables 55, 233.

Wolf, A., Berger, J., Bock, M., Habs, D., Hochadel, B., Kilgus, G., Neureither, G., Schramm, U., Schwalm, D., Szmola, E., Mu.ller, A., Waner, M., Schuch, R., 1991. Z. Phys. D 21 (Suppl.), 569.

Woods, D.T., Shull, J.M., Sarazin, C.L., 1981. Astrophys. J. 249, 399.

Yu, Yan., Seaton, M.J., 1987. J. Phys. B 20, 6409.

Zhang, H.L., Pradhan, A.K., 1997. Phys. Rev. Lett. 78, 195.

Zhang, H.L., Nahar, S.N., Pradhan, A.K., 1999. J. Phys. B 32, 1459.

Zhang, H.L., Nahar, S.N., Pradhan, A.K., 2001. Phys. Rev. A 64, 032719. 